\title{
Transverse momentum dependent gluon distribution within high energy factorization at next-to-leading order
}

\author{
Martin Hentschinski॰* \\ Departamento de Actuaria, Física y Matemáticas, Universidad de las Americas Puebla, \\ Ex-Hacienda Santa Catarina Martir S/N, San Andrs Cholula, 72820 Puebla, Mexico
}

(Received 16 July 2021; accepted 16 August 2021; published 14 September 2021)

\begin{abstract}
We discuss transverse momentum dependent (TMD) gluon distributions within high energy factorization at next-to-leading order in the strong coupling within the framework of Lipatov's high energy effective action. We provide a detailed discussion of both rapidity divergences related to the TMD definition and its soft factor on the one hand, and rapidity divergences due to high energy factorization on the other hand, and discuss common features and differences between Collins-Soper (CS) and Balitsky-Fadin-Kuarev-Lipatov (BFKL) evolution. While we confirm earlier results which state that the unpolarized and linearly polarized gluon TMD agree in the BFKL limit at leading order, we find that both distributions differ, once next-toleading order corrections are being included. Unlike previous results, our framework allows us to recover the complete anomalous dimension associated with the Collins-Soper-Sterman (CSS) evolution of the TMD distribution, including also single-logarithmic terms in the CSS evolution. As an additional result, we provide a definition of $k_{T}$ factorization, i.e., matching of off shell coefficients to collinear factorization at next-to-leading order within high energy factorization and the effective action framework. We furthermore establish a link between the QCD operator definition of the TMD gluon distribution and a previously derived off shell TMD gluon-to-gluon splitting function, which is within the present framework obtained as the real one-loop correction.
\end{abstract}

DOI: 10.1103/PhysRevD.104.054014

\section{INTRODUCTION}

Transverse momentum dependent (TMD) parton distribution functions (PDFs) [1-3] are objects of increased interest, since they allow us to provide a more precise kinematic description of partonic scattering processes already at the leading order (LO) of perturbation theory. This is in particular true, if the observable of interest is not entirely inclusive. In that case, TMD PDFs provide an important advantage over a description based on collinear parton distributions. TMD PDFs arise naturally in processes that are characterized by a hierarchy of scales. With $Q$, the scale of the hard reactions, TMD PDFs are at first defined for the hierarchy $Q \gg q_{T} \gg \Lambda_{\mathrm{QCD}}$ with $q_{T}$, the transverse momentum of the parton, and $\Lambda_{\mathrm{QCD}}$, the QCD characteristic scale of a few hundred MeV. The QCD description of such events gives then rise to the so-called Collins-Soper-Sterman (CSS) [4-6] resummation formalism. A different kinematic hierarchy in which TMD PDFs arise is provided by the

\footnotetext{
*martin.hentschinski@udlap.mx
}

Published by the American Physical Society under the terms of the Creative Commons Attribution 4.0 International license. Further distribution of this work must maintain attribution to the author(s) and the published article's title, journal citation, and DOI. Funded by SCOAP ${ }^{3}$. perturbative Regge or low $x$ limit $\sqrt{s} \gg M \gg \Lambda_{\mathrm{QCD}}$, where $\sqrt{s}$ denotes the center of mass energy of the reaction and $x=M^{2} / s$. While the resulting high energy factorization [7-9] does not primarily address the description of transverse momenta of final states, the ensuing formalism naturally factorizes cross sections into transverse momentum dependent coefficients, so-called impact factors, and transverse momentum dependent Green's function, which summarize logarithms in the center of mass energy. In particular, Balitsky-Fadin-Kuraev-Lipatov (BFKL) evolution [10-15], as well as its nonlinear extensions, keep track of transverse momenta along the evolution chain.

Both kinematic limits have a region of overlap, characterized through the hierarchy $\sqrt{s} \gg M \gg q_{T} \gg \Lambda_{\mathrm{QCD}}$, which is of particular interest due its sensitivity to the emergence of a semihard dynamical scale in the low $x$ limit, the so-called saturation scale [16]. The relation of both frameworks has been explored in a series of publications [17-25] and is currently used for a wide set of phenomenological studies; see, e.g., [26-31]. A somehow orthogonal approach has been put forward in [32-34]: instead of studying the region of overlap of both kinematic regimes, the goal has been to derive TMD evolution kernels, which are meant to achieve a simultaneous resummation of both Dokshitzer-Gribov-Lipatov-Altarelli-Parisi (DGLAP) and BFKL logarithms $[35,36]$. Such an approach seems to be 
of particular interest for Monte Carlo applications such as [37-41]. While currently only real splitting kernels have been derived, which reduce in the regarding limits to the respective real DGLAP and BFKL kernels, the relation to CSS resummation is at the moment less clear; however, the gluon-to-gluon splitting kernel could be shown to reduce to the Ciafaloni-Catani-Fiore-Marchesini kernel $[42,43]$ in the soft limit.

In the following, we aim at solving various open questions related to the previously mentioned studies. In particular, we will give a next-to-leading order (NLO) study with respect to an expansion in the strong coupling constant $\alpha_{s}$ of the gluon TMD in the high energy limit. While a related study has been already presented in [22] within the color-glass-condensate approach, we will investigate this problem within the context of Lipatov's high energy effective action $[44,45]$. Starting with [46-50], the systematic determination of perturbative higher order corrections has been worked out for this framework, while in [51], equivalence with the colorglass-condensate formalism has been demonstrated, including a rederivation of the Balitsky-JIMWLK evolution, see also [52,53] for reviews; for further recent studies based on this framework, see also [54-61]. In [62], a systematic framework for the determination of next-to-leading order corrections at the cross section level has been worked out. For the present study, we will further extend this framework to include asymmetric factorization scale settings as required for a matching to collinear factorization, i.e., $k_{T}$ factorization [8]. While this framework is currently limited to the dilute regime, i.e., two (reggeized) gluon exchange at the level of the cross section, it has the great advantage that it allows us to systematically study different choices of factorization parameter and schemes, which will be of particular use for the further exploration of the relation between BFKL and Collins-Soper (CS) [4,5] evolution initiated in [20]. In particular, we will determine systematically the NLO coefficients that relate the QCD operator definition of the unpolarized and linearly polarized gluon TMD PDF with the unintegrated gluon density of high energy factorization, which in turn will allow us to recover the complete CSS resummation scheme in combination with BFKL evolution, following closely related calculations based on collinear factorization $[63,64]$. We expect our result to be useful for a precise description of final states with small transverse momenta within high energy factorized cross sections, at a similar level of accuracy as descriptions based on collinear factorization.

Another aspect of our result relates to the derivation of TMD splitting kernels in $[32,34]$. While the original derivation was based on a combined implementation of the Curci-Furmanski-Petronzio formalism for the calculation of the collinear splitting functions [65] and the framework of high energy factorization provided by [44], we find in the following that the real contribution to the QCD operator definition of the unpolarized gluon TMD yields precisely the previously derived off shell TMD splitting kernel. Our current study provides therefore a possibility to recover the so far missing virtual corrections to these off shell splitting kernels.

The outline of this paper is as follows. In Sec. II, we give a precise definition of the goal of this paper in more technical terms, in particular the definition of the gluon TMD PDFs; Sec. III contains a brief review of Lipatov's high energy effective action and presents among other details an extension of the framework of [62] to $k_{T}$ factorization. In Sec. IV, we present the results of our NLO calculation, while Sec. V discusses aspects related to the interplay of CS and BFKL evolution. In Sec. VI, we summarize our result and provide an outlook on future research.

\section{THE SETUP OF OUR STUDY}

The starting point of our study is the TMD factorization of a suitable perturbative process. To be specific, we will refer in the following to the transverse momentum distribution of a Higgs boson, as discussed, for instance, in [64]; see also [63]. With $\boldsymbol{p}_{H}$ and $m_{H}$ transverse momentum and the mass of the Higgs boson, this factorization is valid for $\left|\boldsymbol{p}_{H}\right| \ll m_{H}$ and reads

$\frac{d \sigma}{d y_{H} d^{2} \boldsymbol{p}_{H}}=\sigma_{0}(\mu) C_{t}^{2}\left(m_{t}^{2}, \mu\right) H\left(m_{H}^{2}, \mu\right) \int d^{2} \boldsymbol{q}_{a} d^{2} \boldsymbol{q}_{b}(2 \pi)^{2} \delta^{(2)}\left(\boldsymbol{p}_{H}-\boldsymbol{q}_{a}-\boldsymbol{q}_{b}\right) 2 \cdot x_{A} \Gamma_{g / A}^{i j}\left(x_{A}, \zeta_{A} ; \boldsymbol{q}_{a}, \mu\right) \cdot x_{B} \Gamma_{g / B}^{i j}\left(x_{B}, \zeta_{B} ; \boldsymbol{q}_{b}, \mu\right)$,

where $y_{H}=1 / 2 \ln \left(x_{A} / x_{B}\right)$ is the rapidity of the Higgs boson, while $x_{A, B}$ denote the hadron momentum fractions of gluons stemming from hadron $A, B$, respectively, and

$$
\zeta_{A, B}=\left(p_{H}^{ \pm}\right)^{2} e^{\mp 2 y_{c}}=\left(M_{H}^{2}+\boldsymbol{p}_{H}^{2}\right) e^{ \pm 2\left(y_{H}-y_{c}\right)},
$$

where $y_{c}$ denotes the rapidity, which divides soft gluons from hadron $A$ and $B ; \mu$ is the renormalization point of the cross section. To be specific, we consider the scattering of two hadrons with lightlike momenta $p_{A}$ and $p_{B}$, which serve to define the light cone directions,

$$
\left(n^{ \pm}\right)^{\mu}=\frac{2}{\sqrt{s}} p_{A, B}^{\mu}, \quad s=2 p_{A} \cdot p_{B}
$$

which yields the following Sudakov decomposition of a generic four momentum: 


$$
k=k^{+} \frac{n^{-}}{2}+k^{-} \frac{n^{+}}{2}+k_{T}, \quad k^{ \pm}=k \cdot n^{ \pm},
$$

and $n^{ \pm} \cdot k_{T}=0$. Here, $k_{T}$ is the embedding of the Euclidean vector $\boldsymbol{k}$ into Minkowski space, so $k_{T}^{2}=-\boldsymbol{k}^{2}$. For Eq. (1), the top quark is considered to be integrated out, and $C_{t}$ is the corresponding Wilson coefficient; $H$ is the square of the on shell gluon form factor at the timelike momentum transfer $q^{2}=m_{H}^{2}$, with infrared divergences subtracted; see [66]. To a leading order in perturbation theory, they equal one, while the precise NLO expression are not of interest for the following discussion and can be found, for instance, in [64]. $\sigma_{0}(\mu)$ is finally the collinear Born level cross section for the process $g g \rightarrow H$,

$$
\sigma_{0}=\frac{g_{H}^{2} \pi}{8\left(N_{c}^{2}-1\right)}, \quad g_{H}=-\frac{\alpha_{s}(\mu)}{3 \pi v},
$$

with $v \simeq 246 \mathrm{GeV}$ the Higgs vacuum expectation value, and $\alpha_{s}(\mu)$ denotes finally the strong coupling constant at the renormalization point $\mu$. For an unpolarized hadron, the TMD correlator $\Gamma^{i j}, i, j=1,2$ can be further decomposed [67]

$$
\begin{aligned}
\Gamma_{g / B}^{i j}\left(x_{B}, \zeta_{B} ; \boldsymbol{q}, \mu\right)= & -\frac{\delta^{i j}}{2} f_{g / B}\left(x_{B}, \zeta_{B} ; \boldsymbol{q}_{b}, \mu\right) \\
& +\left(\frac{\delta^{i j}}{2}+\frac{\boldsymbol{q}^{i} \boldsymbol{q}^{j}}{\boldsymbol{q}^{2}}\right) h_{g / B}\left(x_{B}, \zeta_{B} ; \boldsymbol{q}_{b}, \mu\right),
\end{aligned}
$$

where $f_{g / B}\left(x_{B}, \zeta_{B} ; \boldsymbol{q}_{b}, \mu\right)$ denotes the unpolarized TMD gluon distriubtion and $h_{g / B}\left(x_{B}, \zeta_{B} ; \boldsymbol{q}_{b}, \mu\right)$ the linearly polarized TMD gluon distribution in an unpolarized hadron. In terms of QCD fields, the TMD PDF is defined as $[63,64]$

$$
\begin{aligned}
x \Gamma_{g / B}^{i j}\left(x_{B}, \zeta_{B} ; \boldsymbol{q}, \mu\right)= & \lim _{\sigma, y_{n} \rightarrow \infty} \int \frac{d \xi^{+} d^{2} \boldsymbol{\xi}}{2(2 \pi)^{3} p_{B}^{-}} e^{i\left(x_{B} p_{B}^{-} \xi^{+} / 2-q \cdot \xi\right)} \tilde{\mathcal{S}}\left(2 y_{c}, \sigma ; \mu, \boldsymbol{\xi}\right) \\
& \left.\cdot\left\langle h\left(p_{B}\right)\left|\operatorname{tr}\left[\left(\mathcal{W}_{\xi}^{n(\sigma)} G^{-i}(\xi)\right)^{\dagger} \mathcal{W}_{0}^{n(\sigma)} G^{-j}(0)\right]\right| h\left(p_{B}\right)\right\rangle\right|_{\xi^{-}=0},
\end{aligned}
$$

where $\tilde{\mathcal{S}}\left(2 y_{c}, \sigma ; \mu, \boldsymbol{\xi}\right)$ denotes the soft factor and $\lim _{\sigma \rightarrow \infty} n(\sigma)=n^{-}$, with $\sigma \rightarrow \infty$ a suitable regulator whose precise implementation will be given in Eq. (48) below. Gauge links are in general given as a combination of a longitudinal and a transverse gauge link [2], where the transverse gauge link is placed at light cone infinity. Working in a covariant gauge, the gauge field at infinity vanishes, and the transverse gauge link therefore equals one. We will therefore in the following not consider the transverse gauge link. The longitudinal gauge link is on the other hand given by

$$
\mathcal{W}_{\xi}^{n}=\mathrm{P} \exp \left(-\frac{g}{2} \int_{-\infty}^{0} d \lambda n \cdot v(\lambda n+\xi)\right),
$$

where $v_{\mu}(x)=-i t^{a} v_{\mu}^{a}(x)$ denotes the gluonic field, and

$$
D_{\mu}=\partial_{\mu}+g v_{\mu}, \quad G^{\mu \nu}=\frac{1}{g}\left[D^{\mu}, D^{\nu}\right]=-i t^{a} G_{a}^{\mu \nu} .
$$

For the soft factor, there exists various prescriptions in the literature; see, e.g., $[2,63,64,68-70]$. To keep the discussion as general as possible, we will consider below the most general soft factor introduced in [2,69],

$$
\tilde{\mathcal{S}}\left(2 y_{c}, \sigma ; \mu, \boldsymbol{\xi}\right)=\sqrt{\frac{\tilde{S}\left(2 y_{c}, 2 y_{n} ; \boldsymbol{\xi}\right)}{\tilde{S}\left(\sigma,-2 y_{c}, ; \boldsymbol{\xi}\right) \tilde{S}\left(\sigma, 2 y_{n} ; \boldsymbol{\xi}\right)}},
$$

with

$$
\begin{aligned}
\tilde{S}\left(y_{1}, y_{2} ; \boldsymbol{\xi}\right)= & \frac{1}{N_{c}^{2}-1} \\
& \cdot\left\langle 0\left|\left(\mathcal{W}_{\boldsymbol{\xi}}^{n_{1}\left(y_{1}\right)}\right)^{\dagger} \mathcal{W}_{\boldsymbol{\xi}}^{n_{2}\left(y_{2}\right)}\left(\mathcal{W}_{\boldsymbol{\xi}}^{n_{2}\left(y_{2}\right)}\right)^{\dagger} \mathcal{W}_{\boldsymbol{\xi}}^{n_{1}\left(y_{1}\right)}\right| 0\right\rangle,
\end{aligned}
$$

where $n_{1,2}\left(y_{1,2}\right)$ are tilted Wilson lines, such that $n_{1}$ is placed at rapidity[71] $y_{1} / 2$ and $n_{2}$ at rapidity $-y_{2} / 2$. For a precise definition of the light cone directions, see Eq. (48) further below. The goal of the following sections is to study this gluon TMD in the high energy limit at next-toleading order. In particular, we will discuss the factorization of this TMD PDF into a perturbative coefficient, the BFKL gluon Green's function, and a hadronic impact factor. The latter two will then form the so-called unintegrated gluon density within high energy factorization. Our study is limited to the exchange of two reggeized gluons. It is known from various studies that the gluon TMD also receives corrections due to the exchange of multiple reggeized gluons, which are of importance to take into account corrections due to high gluon densities and their possible saturation; see, e.g., [17,24,27,29,72]. While these are very interesting questions-in particular since they can provide modifications of the region of very small transverse momenta due to the emergence of a saturation scale-we do not consider these effects in the following. Instead, great care will be taken to provide a complete discussion of various factorization scales and parameters both due to factorization in the soft limit and 
the high energy limit, as well as UV renormalization. The study of these effects is somehow more straightforward, if the observable is restricted to two reggeized gluon exchange, which is the reason why we focus on this limit in the following. The obtained results may then be generalized at a later stage to the case of multiple reggeized gluon exchange.

Another motivation for this study is to link the above gluon TMD to the TMD splitting kernels derived in [32,34]. Below, we will demonstrate that the TMD gluon-to-gluon splitting of [34] arises directly from the real contributions to the one-loop coefficient. We believe that this is a very interesting result, since it allows us to connect the framework of real TMD splitting kernels to the above operator definitions of TMD PDFs.

\section{THE HIGH-ENERGY EFFECTIVE ACTION}

Since the current study requires a small but important generalization in comparison to the framework presented in [29], we begin our study with a short review of the high energy effective action and the resulting calculational framework for NLO calculations. Our treatment of high energy factorization is based on Lipatov's high energy effective action [44]. Within this framework, QCD amplitudes in the high energy limit are decomposed into gauge invariant subamplitudes, which are localized in rapidity space and describe the coupling of quarks $(\psi)$, gluon $\left(v_{\mu}\right)$, and ghost $(\phi)$ fields to a new degree of freedom, the reggeized gluon field $A_{ \pm}(x)$. The latter is introduced as a convenient tool to reconstruct the complete QCD amplitudes in the high energy limit out of the subamplitudes restricted to small rapidity intervals. Lipatov's effective action is then obtained by adding an induced term $S_{\text {ind }}$ to the QCD action $S_{\mathrm{QCD}}$,

$$
S_{\text {eff }}=S_{\mathrm{QCD}}+S_{\text {ind }},
$$

where the induced term $S_{\text {ind }}$ describes the coupling of the gluonic field $v_{\mu}=-i t^{a} v_{\mu}^{a}(x)$ to the reggeized gluon field $A_{ \pm}(x)=-i t^{a} A_{ \pm}^{a}(x)$. High energy factorized amplitudes reveal strong ordering in plus and minus components of momenta, which is reflected in the following kinematic constraint obeyed by the reggeized gluon field:

$$
\partial_{+} A_{-}(x)=0=\partial_{-} A_{+}(x) .
$$

Even though the reggeized gluon field is charged under the QCD gauge group $\mathrm{SU}\left(N_{c}\right)$, it is invariant under local gauge transformation $\delta A_{ \pm}=0$. Its kinetic term and the gauge invariant coupling to the QCD gluon field are contained in the induced term,

$$
\begin{aligned}
S_{\text {ind }}= & \int \mathrm{d}^{4} x \operatorname{tr}\left[\left(W_{-}[v(x)]-A_{-}(x)\right) \partial_{\perp}^{2} A_{+}(x)\right] \\
& +\operatorname{tr}\left[\left(W_{+}[v(x)]-A_{+}(x)\right) \partial_{\perp}^{2} A_{-}(x)\right],
\end{aligned}
$$

with

$W_{ \pm}[v(x)]=v_{ \pm}(x) \frac{1}{D_{ \pm}} \partial_{ \pm}, \quad D_{ \pm}=\partial_{ \pm}+g v_{ \pm}(x)$.

For a more in depth discussion of the effective action, we refer to the reviews $[52,53]$. Due to the induced term in Eq. (12), the Feynman rules of the effective action comprise, apart from the usual QCD Feynman rules, the propagator of the reggeized gluon and an infinite number of so-called induced vertices. Vertices and propagators needed for the current study are collected in Fig. 1. Determination of NLO corrections using this effective action approach has been addressed recently in series of publications [46-50]. For a discussion of the analogous high energy effective for flavor exchange [73] at NLO, see, e.g., [74-76].

\section{A. Determination of NLO coefficients}

The framework for the determination of NLO corrections has been established in [62] within the determination of the NLO forward Higgs production coefficient in the infinite top mass limit. We will therefore frequently refer to the process,

$$
\operatorname{gluon}\left(p_{a}\right)+\operatorname{quark}\left(p_{b}\right) \rightarrow \operatorname{Higgs}\left(p_{H}\right)+X,
$$

as an example process in the following, where we further assume that the particles in the fragmentation region of the scattering particles are widely separated in rapidity. The partonic impact factor of the quark with momentum $p_{b}$ will be later on replaced by the hadronic impact factor, which

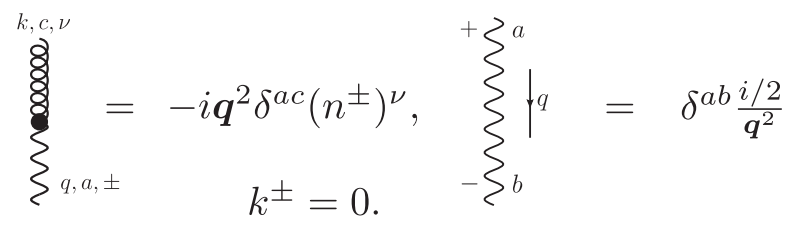

(a) (b)

FIG. 1. Feynman rules for the lowest-order effective vertices of the effective action. Wavy lines denote reggeized fields and curly lines gluons. 
forms together with the BFKL Green's function, the unintegrated gluon density. As in [62], we will consider matrix elements normalized to match corresponding collinear matrix elements for vanishing virtuality of the reggeized gluon state. We therefore have

$$
\overline{\left|\mathcal{A}_{a r^{+} \rightarrow X_{a}^{(n)}}\right|^{2}}=\frac{\left(k^{-}\right)^{2}}{4 \boldsymbol{k}^{2}} \overline{\left|\mathcal{M}_{a r^{+} \rightarrow X_{a}^{(n)}}\right|^{2}}
$$

where we average over incoming parton color as well as the color of the reggeized gluon and sum over the color of produced particles; $X_{a}^{(n)}$ denotes the $n$-particle system produced in the regarding fragmentation region. With

$$
\begin{aligned}
d \Gamma^{(n)} & =(2 \pi)^{d} \delta^{d}\left(p_{a}+k-\sum_{j=1}^{n} p_{j}\right) d \Phi^{(n)}, \\
d \Phi^{(n)} & =\prod_{j=1}^{n} \frac{d^{d} p_{j}}{(2 \pi)^{d-1}} \delta_{+}\left(p_{j}^{2}-m_{j}^{2}\right),
\end{aligned}
$$

we arrive at the following definition of an off shell partonic cross section $d \hat{\sigma}_{a+}$ and the corresponding impact factor $\hat{h}^{k_{T}}(\boldsymbol{k})$ :

$$
\begin{aligned}
d \hat{\sigma}_{a+} & =\frac{\left.\overline{\mid \mathcal{A}_{a r^{+} \rightarrow X_{a}^{(n)}}}\right|^{2}}{2 p_{a}^{+} k^{-}} d \Gamma^{(n)}, \\
\hat{h}^{k_{T}}(\boldsymbol{k}) & =\int \frac{d k^{-}}{k^{-}} d \hat{\sigma}_{a+} .
\end{aligned}
$$

Note that this impact factor can in principle be arbitrarily differential, as far as the formulation of high energy factorization is concerned; for a corresponding definition of the other impact factor, we refer to [62]. The above expression is subject to so-called rapidity divergences, which are understood to be regulated through lower cutoffs

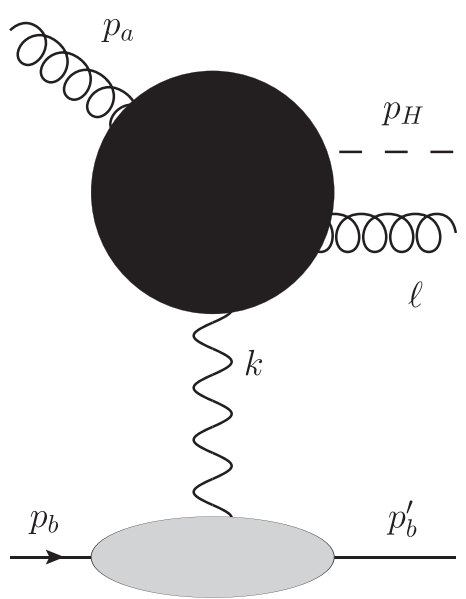

on the rapidity of all particles, $\eta_{i}>-\rho / 2$ with $\rho \rightarrow \infty$ and $i=1, \ldots, n$ for $n$ the number of particles produced in the fragmentation region of the initial parton $a$. For virtual corrections, the regularization is achieved through tilting light cone directions of the high energy effective action,

$$
n^{+} \rightarrow n^{+}+e^{-\rho} n^{-}, \quad \rho \rightarrow \infty .
$$

Below we will also comment on the possibility to regularize rapidity divergences through tilting light cone direction also in the case of real corrections; see Sec. III D.

As shown through explicit results [46,48-50,62], impact factors contain beyond leading order configurations, which reproduce factorized contributions with internal reggeized gluon exchange. It is therefore necessary to subtract these contributions; see also Fig. 2. To this end, one defines the bare one-loop two-reggeized-gluon Green's function $G_{B}\left(\boldsymbol{k}_{1}, \boldsymbol{k}_{2}\right)$ as well as the impact factors through the following perturbative expansion:

$$
\begin{aligned}
G_{B}\left(\boldsymbol{k}_{1}, \boldsymbol{k}_{2} ; \rho\right) & =\delta^{(2+2 \epsilon)}\left(\boldsymbol{k}_{1}+\boldsymbol{k}_{2}\right)+G_{B}^{(1)}\left(\boldsymbol{k}_{1}, \boldsymbol{k}_{2} ; \rho\right)+\cdots \\
h_{a}(\boldsymbol{k}, \rho) & =h_{a}^{(0)}(\boldsymbol{k})+h_{a}^{(1)}(\boldsymbol{k}, \rho)+\cdots
\end{aligned}
$$

Using the following convolution convention:

$$
[f \otimes g]\left(\boldsymbol{k}_{1}, \boldsymbol{k}_{2}\right) \equiv \int d^{2+2 \epsilon} \boldsymbol{q} f\left(\boldsymbol{k}_{1}, \boldsymbol{q}\right) g\left(\boldsymbol{q}, \boldsymbol{k}_{2}\right),
$$

we then define the following subtracted bare NLO coefficient,

$$
\begin{aligned}
C_{a, B}^{(1)}(\boldsymbol{k}, \rho)= & h_{a}^{(0)}(\boldsymbol{k})+h_{a}^{(1)}(\boldsymbol{k}, \rho) \\
& -\left[h_{a}^{(0)} \otimes G_{B}^{(1)}(\rho)\right](\boldsymbol{k}),
\end{aligned}
$$

and [77]

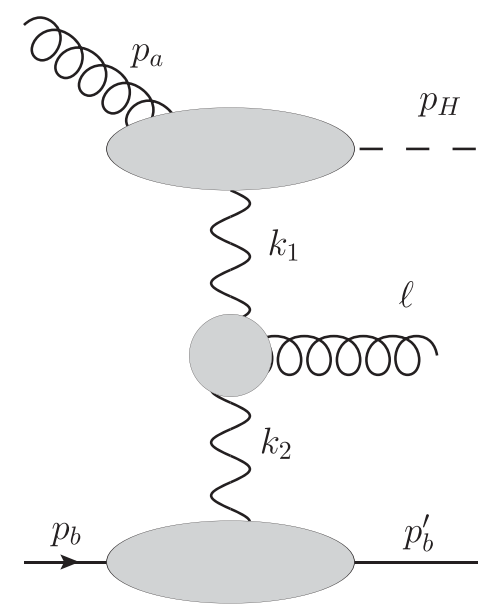

FIG. 2. NLO correction to forward Higgs production (left) and the factorized matrix element with internal reggeized gluon exchange (right). The NLO correction contains the factorized contribution, which need to be subtracted from the former. 


$$
\begin{aligned}
d \sigma_{a b}^{\mathrm{NLO}}= & \frac{1}{\pi^{1+\epsilon}}\left[C_{a, B}^{k_{T}}(\rho) \otimes G_{B}(\rho) \otimes C_{b, B}^{(u g d)}(\rho)\right] \\
& + \text { terms beyond NLO, }
\end{aligned}
$$

where we added the superscripts " $k_{T}$ " and " $u g d "$ to indicate that the impact factor of the particle with momentum $p_{a, b}$ refers to the hard event and the unintegrated gluon distribution, respectively. While rapidity divergences cancel for the above expression, its elements still depend on the regulator. As a next step, we therefore define a renormalized Green's function $G_{R}$ through

$$
\begin{aligned}
G_{B}\left(\boldsymbol{k}_{1}, \boldsymbol{k}_{2} ; \rho\right)= & {\left[Z^{+}\left(\frac{\rho}{2}-\eta_{a}\right) \otimes G_{R}\left(\eta_{a}-\eta_{b}\right)\right.} \\
& \left.\otimes Z^{-}\left(\frac{\rho}{2}+\eta_{b}\right)\right]\left(\boldsymbol{k}_{1}, \boldsymbol{k}_{2}\right),
\end{aligned}
$$

which yields

$$
d \sigma_{a b}^{\mathrm{NLO}}=\left[C_{a, R}\left(\eta_{a}\right) \otimes G_{R}\left(\eta_{a}, \eta_{b}\right) \otimes C_{b, R}\left(\eta_{b}\right)\right],
$$

where

$$
\begin{aligned}
& C_{a, R}\left(\eta_{a} ; \boldsymbol{k}_{1}\right) \equiv\left[C_{a}(\rho) \otimes Z^{+}\left(\frac{\rho}{2}-\eta_{a}\right)\right]\left(\boldsymbol{k}_{1}\right), \\
& C_{b, R}\left(\eta_{b} ; \boldsymbol{k}_{2}\right) \equiv\left[Z^{+}\left(\frac{\rho}{2}+\eta_{b}\right) \otimes C_{b}(\rho)\right]\left(\boldsymbol{k}_{2}\right) .
\end{aligned}
$$

The transition functions $Z^{ \pm}$have a twofold purpose: they both serve to cancel $\rho$-dependent terms between impact factors and Green's function and allow us to define the BFKL kernel. In particular,

$$
\begin{aligned}
& \frac{d}{d \hat{\rho}} Z^{+}(\hat{\rho} ; \boldsymbol{k}, \boldsymbol{q})=\left[Z^{+}(\hat{\rho}) \otimes K_{\mathrm{BFKL}}\right](\boldsymbol{k}, \boldsymbol{q}), \\
& \frac{d}{d \hat{\rho}} Z^{-}(\hat{\rho} ; \boldsymbol{k}, \boldsymbol{q})=\left[K_{\mathrm{BFKL}} \otimes Z^{-}(\hat{\rho})\right](\boldsymbol{k}, \boldsymbol{q}),
\end{aligned}
$$

where

$$
K_{\mathrm{BFKL}}(\boldsymbol{k}, \boldsymbol{q})=K^{(1)}(\boldsymbol{k}, \boldsymbol{q})+K^{(2)}(\boldsymbol{k}, \boldsymbol{q})+\cdots
$$

\section{B. Transition function and finite terms}

In the following, we generalize the treatment given in [62] through including as well the most general finite contribution into our discussion. The need to include finite contribution into this transition factors has been first realized in the determination of the two-loop gluon Regge trajectory in [48-50], where both divergent and finite terms could be simply taken to exponentiate, since one is dealing with one-reggeized gluon exchange contributions only. A suitable generalization, which both reduces to the exponential ansatz of [50] and obeys Eq. (28), is then given by

$$
\begin{aligned}
& Z^{+}(\hat{\rho} ; \boldsymbol{k}, \boldsymbol{q})=\delta^{(2+2 \epsilon)}(\boldsymbol{k}-\boldsymbol{q})+\hat{\rho} K_{\mathrm{BFKL}}(\boldsymbol{k}, \boldsymbol{q})+\frac{\hat{\rho}^{2}}{2} K_{\mathrm{BFKL}} \otimes K_{\mathrm{BFKL}}(\boldsymbol{k}, \boldsymbol{q})+f^{+} \otimes \hat{\rho} K_{\mathrm{BFKL}}(\boldsymbol{k}, \boldsymbol{q})+f^{+}(\boldsymbol{k}, \boldsymbol{q})+\frac{f^{+} \otimes f^{+}(\boldsymbol{k}, \boldsymbol{q})}{2} \ldots, \\
& Z^{-}(\hat{\rho} ; \boldsymbol{k}, \boldsymbol{q})=\delta^{(2+2 \epsilon)}(\boldsymbol{k}-\boldsymbol{q})+\hat{\rho} K_{\mathrm{BFKL}}(\boldsymbol{k}, \boldsymbol{q})+\frac{\hat{\rho}^{2}}{2} K_{\mathrm{BFKL}} \otimes K_{\mathrm{BFKL}}(\boldsymbol{k}, \boldsymbol{q})+\hat{\rho} K_{\mathrm{BFKL}} \otimes f^{-}(\boldsymbol{k}, \boldsymbol{q})+f^{-}(\boldsymbol{k}, \boldsymbol{q})+\frac{f^{-} \otimes f^{-}(\boldsymbol{k}, \boldsymbol{q})}{2} \ldots,
\end{aligned}
$$

which is sufficient for a discussion up to NLO accuracy. As we will see in the following, these finite contributions serve a twofold purpose. At first, they remove a potential finite contribution in the bare Green's function,

$$
\begin{aligned}
G_{B}^{(1)}\left(\boldsymbol{k}_{1}, \boldsymbol{k}_{2} ; \rho\right)= & \frac{\alpha_{s} C_{A} \rho}{\pi}\left(\frac{1}{\pi_{\epsilon}\left(\boldsymbol{k}_{1}-\boldsymbol{k}_{2}\right)^{2}}-\frac{1}{\epsilon}\left(\frac{\boldsymbol{k}_{1}^{2}}{\mu^{2}}\right)^{\epsilon} \delta^{(2+2 \epsilon)}\left(\boldsymbol{k}_{1}-\boldsymbol{k}_{2}\right)\right) \\
& -\frac{\alpha_{s}}{2 \pi}\left(\frac{\boldsymbol{k}_{1}^{2}}{\mu^{2}}\right)^{\epsilon}\left(\frac{5 C_{A}-2 n_{f}}{3 \epsilon}-\frac{31 C_{A}}{9}+\frac{10 n_{f}}{9}\right) \delta^{(2+2 \epsilon)}\left(\boldsymbol{k}_{1}-\boldsymbol{k}_{2}\right),
\end{aligned}
$$

where

$$
\pi_{\epsilon} \equiv \pi^{1+\epsilon} \Gamma(1-\epsilon) \mu^{2 \epsilon}, \quad \alpha_{s}=\frac{g^{2} \Gamma(1-\epsilon) \mu^{2 \epsilon}}{(4 \pi)^{1+\epsilon}},
$$

which then yields directly the one-loop BFKL kernel,

$$
K^{(1)}\left(\boldsymbol{k}_{1}, \boldsymbol{k}_{2}\right)=\frac{\alpha_{s} C_{A}}{\pi}\left[\frac{1}{\pi_{\epsilon}\left(\boldsymbol{k}_{1}-\boldsymbol{k}_{2}\right)^{2}}-\frac{1}{\epsilon}\left(\frac{\boldsymbol{k}_{1}^{2}}{\mu^{2}}\right)^{\epsilon} \delta^{(2+2 \epsilon)}\left(\boldsymbol{k}_{1}-\boldsymbol{k}_{2}\right)\right] .
$$

To keep the treatment as general as possible, the finite contribution is on the other hand then split up into two terms, 


$$
f^{ \pm,(1)}\left(\boldsymbol{k}_{1}, \boldsymbol{k}_{2}\right)=\tilde{f}^{ \pm,(1)}\left(\boldsymbol{k}_{1}, \boldsymbol{k}_{2}\right)+\bar{f}^{ \pm,(1)}\left(\boldsymbol{k}_{1}, \boldsymbol{k}_{2}\right) .
$$

The contribution $\tilde{f}^{ \pm}$is used to transfer finite terms contained in the Green's function to the impact factors. We take, in the following, this function to be identical for both plus and minus direction $\tilde{f}^{ \pm,(1)}\left(\boldsymbol{k}_{1}, \boldsymbol{k}_{2}\right)=\tilde{f}^{(1)}\left(\boldsymbol{k}_{1}, \boldsymbol{k}_{2}\right)$. Indeed, since we are essentially dealing with contributions due to the gluon polarization tensor in the high energy limit, such a symmetric treatment appears to be the appropriate one. One finds

$$
\begin{aligned}
\tilde{f}^{(1)}\left(\boldsymbol{k}_{1}, \boldsymbol{k}_{2}\right)= & \frac{\alpha_{s}}{4 \pi}\left[-\frac{5 C_{A}-2 n_{f}}{3 \epsilon}\left(\frac{\boldsymbol{k}_{1}^{2}}{\mu^{2}}\right)^{\epsilon}\right. \\
& \left.+\frac{31 C_{A}-10 n_{f}}{9}\right] \delta^{(2+2 \epsilon)}\left(\boldsymbol{k}_{1}-\boldsymbol{k}_{2}\right)+\mathcal{O}(\epsilon) .
\end{aligned}
$$

The second contribution $\bar{f}^{ \pm}$was absent in the discussion of [62]. It needs to satisfy to one-loop the following requirement, to ensure absence of finite terms in the Green's function:

$$
\bar{f}^{+,(1)}\left(\boldsymbol{k}_{1}, \boldsymbol{k}_{2}\right)=-\bar{f}^{-,(1)}\left(\boldsymbol{k}_{1}, \boldsymbol{k}_{2}\right) \equiv \bar{f}^{(1)}\left(\boldsymbol{k}_{1}, \boldsymbol{k}_{2}\right) .
$$

Note that similar constraints can be imposed on higher order contributions to the functions $\bar{f}^{ \pm}$to achieve a Green's function without finite contributions at higher orders. Including all contributions, one finally arrives at following expression for the NLO coefficient:

$$
\begin{aligned}
C_{R, i}^{\mathrm{NLO}}(\boldsymbol{k})= & h_{a}^{(0)}(\boldsymbol{k})+h_{a}^{(1)}(\boldsymbol{k})+h_{a}^{(0)} \\
& \otimes\left[\left(-\frac{\rho}{2}-s_{i} \eta_{i}\right) K^{(1)}+s_{i} \bar{f}^{(1)}-\tilde{f}^{(1)}\right](\boldsymbol{k}) \\
& i=a, b, \quad s_{a, b}= \pm,
\end{aligned}
$$

where $K^{(1)}\left(\boldsymbol{k}_{1}, \boldsymbol{k}_{2}\right)$ and $\tilde{f}^{(1)}\left(\boldsymbol{k}_{1}, \boldsymbol{k}_{2}\right)$ are given in Eq. (33) and Eq. (35), respectively. The function $\bar{f}^{(1)}\left(\boldsymbol{k}_{1}, \boldsymbol{k}_{2}\right)$ as well as the evolution parameters $\eta_{a, b}$ are on the other hand still undetermined. They are in principle arbitrary, but should be chosen such that both impact factors are free of large logarithms.

\section{Scale setting for $k_{T}$ factorization}

The parameters $\eta_{a, b}$ as well as the function $\bar{f}$ are at first arbitrary; the former define through the combination $\eta_{a}-$ $\eta_{b}$ the evolution parameter of the two reggeized gluon Green's function $G_{R}$; see also the related discussion in $[53,62]$. Usually, it is necessary to chose these parameters such that the next-to-leading order corrections to impact factors are under perturbative control and that large logarithms in the center of mass energy are resumed through the two reggeized gluon Green's function. Within the $k_{T}$-factorization setup, one of the impact factors, e.g., the coefficient $C_{R, b}\left(\eta_{b}, \boldsymbol{k}_{2}\right)$ in our example, is to be replaced by the hadronic impact factor, which then builds together with the BFKL Green's function the unintegrated gluon density. Even though the hadronic impact factor is naturally a nonperturbative object, at the very least for transverse momenta $\left|\boldsymbol{k}_{2}\right| \gtrsim 1.5 \mathrm{GeV}$, it must have an expansion in terms of collinear parton distribution functions and corresponding collinear coefficients; see e.g., [78]. In order to have a complete matching of the resulting expression for the unintegrated gluon density to the collinear gluon distribution in the double-logarithmic limit, it is natural to chose the evolution parameter $\eta_{a}-\eta_{b}$ to coincide with the fraction of the hadronic momentum carried on by the gluon $x \simeq M_{a}^{2} / s$ with $M_{a}$ the invariant mass of the system produced in the fragmentation region [79]. Note that at NLO, an asymmetric scale choice, i.e., choosing the reference scale of the center-of-mass energy $\sqrt{s}$ to be of the order of a typical scale of one of the impact factors, leads to a modification of the NLO BFKL kernel; see, e.g., $[14,80]$. To repeat this exercise within the context of the high energy effective action, we reconsider Eq. (27), but focus now on the factorization parameters $\eta_{a, b}$ and the finite terms introduced above,

$$
\begin{aligned}
C_{R, a}^{\mathrm{NLO}}\left(\boldsymbol{k}_{1}, M_{a}, \eta_{a}, \bar{f}^{(1)}\right)= & \hat{C}_{R, a}^{\mathrm{NLO}}\left(\boldsymbol{k}_{1}, M_{a}\right)-\left[h_{a}^{(0)}\left(M_{a}\right)\right. \\
& \left.\otimes\left(\eta_{a} K^{(1)}-\bar{f}^{(1)}\right)\right]\left(\boldsymbol{k}_{1}\right) \\
C_{R, b}^{\mathrm{NLO}}\left(\boldsymbol{k}_{2}, M_{b}, \eta_{b}, \bar{f}^{(1)}\right)= & \hat{C}_{R, b}^{\mathrm{NLO}}\left(\boldsymbol{k}_{2}, M_{a}\right)+\left[h_{b}^{(0)}\left(M_{a}\right)\right. \\
& \left.\otimes\left(\eta_{b} K^{(1)}-\bar{f}^{(1)}\right)\right]\left(\boldsymbol{k}_{2}\right),
\end{aligned}
$$

where $M_{a, b}$ is the invariant mass of the produced final state corresponding to each of the impact factors and $\hat{C}_{R}$ collects all terms which are independent of both $\eta_{a, b}$ and $\bar{f}$. To equal the evolution parameter of the Green's function with the hadron momentum fraction carried on by the reggeized gluon entering the impact factor $C_{R, a}$, we set

$$
\begin{aligned}
\eta_{a} & =\ln \frac{M_{0}}{k^{-}}, \quad \eta_{b}=\ln \frac{M}{p_{b}^{-}}, \\
\eta_{a}-\eta_{b} & =\ln \frac{p_{b}^{-}}{k^{-}}+\ln \frac{M_{0}}{M}=\ln \frac{x_{0}}{x_{g}},
\end{aligned}
$$

where $M, M_{0}$ are so far an unspecified reference scale and $x_{0}=M_{0} / M$ is a parameter of order one, which allows us to estimate the scale uncertainty associated with high energy factorization. In the following, we chose $M$ to be of the order of $M_{a}$, i.e., the hard scale. While this is a natural choice for the hard impact factor, it introduces the same scale into the hadronic impact factor, characterized in general by small transverse momenta. We therefore find in the perturbative region of the hadronic impact factor a large collinear logarithm, which at first spoils the 
convergence of the perturbative expansion. This logarithm can however be absorbed into the $\bar{f}$ function through setting,

$$
\bar{f}_{k_{T}}^{(1)}\left(\boldsymbol{k}_{2}, \boldsymbol{q}_{2}\right) \equiv \ln \frac{M}{\left|\boldsymbol{k}_{2}\right|} K^{(1)}\left(\boldsymbol{k}_{2}, \boldsymbol{q}_{2}\right)
$$

which eliminates the logarithm in $M$ from the hadronic impact factor. Note that the choice of $\left|\boldsymbol{k}_{2}\right|$ as the relative scale is somewhat arbitrary, and other choices are equally possible; see, e.g., [78]. It is interesting to compare this situation to the case where the parameters $\eta_{a, b}$ are identified with the rapidities of the system produced in the regarding fragmentation region, $\eta_{a}=\ln p_{a}^{+} / M_{a}$, $\eta_{b}=\ln M_{b} / p_{b}^{-}$, with $\bar{f}^{(1)}=0$. One finds

$$
\begin{aligned}
& C_{R, b}^{\mathrm{NLO}}\left(\boldsymbol{k}_{2}, M_{b}, \ln \frac{M}{p_{b}^{-}}, \bar{f}_{k_{T}}^{(1)}\right)=C_{R, b}^{\mathrm{NLO}}\left(\boldsymbol{k}_{2}, M_{b}, \ln \frac{M_{b}}{p_{b}^{-}}, 0\right) \\
& -\ln \frac{M_{b}}{\left|\boldsymbol{k}_{2}\right|} \int d^{2+2 \epsilon} \boldsymbol{q}_{2}\left[K^{(1)}\left(\boldsymbol{k}_{2}, \boldsymbol{q}_{2}\right) h_{b}^{(0)}\left(M_{b}, \boldsymbol{q}_{2}\right)\right], \\
& C_{R, a}^{\mathrm{NLO}}\left(\boldsymbol{k}_{1}, M_{a}, \ln \frac{M_{0}}{k^{-}}, \bar{f}_{k_{T}}^{(1)}\right)=C_{R, a}^{\mathrm{NLO}}\left(\boldsymbol{k}_{1}, M_{a}, \ln \frac{p_{a}^{+}}{M_{a}}, 0\right) \\
& \quad+\int d^{2+2 \epsilon} \boldsymbol{q}_{1}\left[h_{a}^{(0)}\left(M_{a}, \boldsymbol{q}_{a}\right) \cdot \ln \frac{M_{a}}{x_{0}\left|\boldsymbol{q}_{1}\right|} \cdot K^{(1)}\left(\boldsymbol{q}_{1}, \boldsymbol{k}_{1}\right)\right],
\end{aligned}
$$

which allows us to verify that the presented treatment agrees-after setting $x_{0}=1$-with the one derived in [80], based on a study of ladder diagrams within the quasimulti-Regge-kinematics in the context of the definition of the NLO inclusive jet vertex. For the NLO BFKL kernel, one finally obtains the following contribution:

$$
\begin{aligned}
K_{k_{T}}^{(2)}\left(\boldsymbol{k}_{1}, \boldsymbol{k}_{2}\right)= & K^{(2)}\left(\boldsymbol{k}_{1}, \boldsymbol{k}_{2}\right) \\
& -\frac{1}{2} \int d^{2} \boldsymbol{k} \ln \frac{\boldsymbol{k}^{2}}{\boldsymbol{k}_{1}^{2}} K^{(1)}\left(\boldsymbol{k}_{1}, \boldsymbol{k}\right) K^{(1)}\left(\boldsymbol{k}, \boldsymbol{k}_{2}\right),
\end{aligned}
$$

which is independent of the parameter $x_{0}$, and where $K^{(2)}$ denotes the NLO BFKL kernel if $\eta_{a, b}$ is identified with the rapidities of the external particles with $\bar{f}^{(1)}=0$. The above expression is in agreement with [80] and [14]. A more detailed discussion of possible choices of the function $\bar{f}^{(1)}$ will be presented elsewhere.

Summing up we have the general definition of the unintegrated gluon density,

$$
\begin{aligned}
\mathcal{G} & \left(\Delta \eta_{a b}, \eta_{b}, \boldsymbol{k}, \bar{f}^{(1)}\right) \\
& =\int d^{2+2 \epsilon} \boldsymbol{q} G_{R}\left(\Delta \eta_{a b}, \boldsymbol{k}, \boldsymbol{q}\right) C_{R, b}^{\mathrm{NLO}}\left(\boldsymbol{q}, \eta_{b}, \bar{f}^{(1)}\right),
\end{aligned}
$$

where we suppressed the dependence on the invariant mass $M_{b}$ since the latter can in general be expressed in terms of the transverse momentum. The $k_{T}$-factorization scheme fixes then $\bar{f}^{(1)}$ through Eq. (40), while $\Delta \eta_{a b}=\eta_{a}-\eta_{b}$ is set to $\Delta \eta_{a b}^{k_{T}} \equiv \ln 1 / x_{g}$. The high energy factorized cross section is then obtained as

$$
\begin{aligned}
d \sigma_{A B}= & \int \frac{d^{2+2 \epsilon} \boldsymbol{k}}{\pi^{1+\epsilon}} d C_{R, a}^{\mathrm{NLO}}\left(\boldsymbol{k}, M_{a}, \eta_{a}, \bar{f}^{(1)}\right) \\
& \times \mathcal{G}\left(\Delta \eta_{a b}, \eta_{b}, \boldsymbol{k}, \bar{f}^{(1)}\right),
\end{aligned}
$$

where " $A$ " might either denote a parton $a$, a partonic impact factor convoluted with a parton distribution function of a hadron $A$ or a colorless initial state which allows for a perturbative treatment. Concluding, we remark that in [81] a definition of the unintegrated gluon density has been proposed in terms of a operator definition with the high energy gluonic field in light cone gauge, which requires the inclusion of both so-called two, three, and four body contributions. While an interpretation of such contributions in terms of induced vertices Fig. 1 of the high energy effective action appears to be possible, the precise relation remains unclear.

\section{Regularization of rapidity divergences}

As pointed out in the above discussion, high energy factorized matrix elements are subject to so-called rapidity divergences. While they cancel at the level of observables after subtraction of factorizing contribution and use of the transition function, intermediate results beyond leading order require a regulator in order to arrive at well-defined matrix elements. While for real production a cutoff on the rapidity of produced particles provides a natural way to regulate such divergences, a consistent regularization is more difficult to achieve in the case of virtual diagrams. As already pointed out in Sec. III A, a suitable way to regulate these divergences in the case of virtual corrections is to tilt the light cone directions of the high energy effective action away from the light cone; see Eq. (20). Note that from a formal point of view this a very attractive way of regularizing rapidity divergences, since gauge invariance of the high energy effective action does not depend on the property $n^{ \pm 2}=0$; tilting light cone directions provides therefore a gauge invariant regulator, similar to dimensional regularization. Nevertheless, the current treatment, see, e.g., [46,48,62], is somewhat unsatisfactory, since it treats real (cutoff) and virtual (tilting) corrections on somewhat different grounds. At the same time, tilting light cone directions is also a frequently used regulator for the determination of the oneloop corrections to TMD PDFs within collinear factorization; see, e.g., [2,63] and references therein, which is being use for both real and virtual corrections. It seems therefore natural to regulate rapidity divergences through tilting light cone directions also in the case of real corrections. 
From a technical point, this does not imply any major complications. However, the real part of the one-loop Green's function Eq. (31) would receive a finite correction, which in covariant Feynman gauge is related to the square of the induced diagrams (last two diagrams in Fig. 3). As can be seen already at the level of diagrams, such contributions arise as well for the corresponding impact factors, which contain an identical diagram once calculating the correction due to the emission of an additional real gluon. As a consequence, it is straightforward to show that the corresponding contributions cancel, once the subtracted impact factor and central contribution are combined. Moreover, such a contribution may be easily absorbed into a generalized version of the function $\tilde{f}^{(1)}$, Eq. (35). While including such contributions does not provide any substantial complication, one deals in that case with an entirely spurious contribution, which merely arises due to our choice of our regulator and which has no physical meaning. It seems therefore natural to employ a regulator which avoids such a contribution altogether, at least at one-loop. The modified regulator is essentially identical to the previously used tilted light cone vectors, while the tilted elements are taken now to be complex; i.e., we will use in the following:

$$
n^{ \pm} \rightarrow n^{b, a}=n^{ \pm}+i e^{-\rho} n^{\mp}, \quad \rho \in \mathbb{R} .
$$

As a consequence, one has for virtual corrections,

$$
n^{a, b 2}=-4 e^{-\rho}, \quad n^{a} \cdot n^{b}=2\left(1-e^{-2 \rho}\right),
$$

while real corrections yields

$$
\left|n^{a, b}\right|^{2}=0, \quad n^{a} \cdot\left(n^{b}\right)^{*}+\text { c.c. }=4\left(1+e^{-2 \rho}\right) .
$$

The spurious self-energy like contributions are therefore absent. The only disadvantage of this method is that terms of the form $\ln \left(n^{a, b 2}\right)$ in virtual corrections can give rise to undesired imaginary parts due to spacelike $n^{a, b 2}$. While at the cross section level such imaginary parts cancel naturally, if one limits oneself to NLO corrections, a consistent treatment of such a contribution at the amplitude level would require to absorb this imaginary part into the parameter $\rho$, e.g., through a suitable replacement $\rho \rightarrow \tilde{\rho}=$ $\rho-i \pi / 2$ etc., in the transition functions.

In the following calculation, we will meet rapidity divergences that originate both from high energy factorization and the QCD operator definition of the TMD gluon distribution and the corresponding soft factor, which we will consistently regulate through the tilting as described in Eq. (45), while we reserve the use of the regulator $\rho \rightarrow \infty$ for rapidity divergences due to high energy factorization. To be specific, we define in the following the tilted Wilson lines of the TMD definition Eq. (7) and Eq. (11) as

$$
\begin{aligned}
n_{1,2}\left(y_{1,2}\right) & =n^{\mp}+i e^{-y_{1,2} n^{ \pm},} \\
n(\sigma) & =n^{-}+i e^{-\sigma} n^{+} .
\end{aligned}
$$

\section{DETERMINATION OF THE GLUON TMD}

The goal of the following section is to determine the gluon TMD Eq. (7) within high energy factorization; i.e., we aim at the determination of the following coefficient $C_{g g^{*}}$, implicitly defined through

$$
\begin{aligned}
& f_{g}\left(\eta_{a}, \eta_{b}, y_{c}, \zeta_{B}, \boldsymbol{q}, \mu\right) \\
& \quad=\int \frac{d^{2} \boldsymbol{k}}{\pi} C_{g g^{*}}^{f}\left(\zeta_{B}, y_{c}, \eta_{a}, \boldsymbol{q}, \boldsymbol{k}, \mu\right) \mathcal{G}\left(\Delta \eta_{a b}, \eta_{b} ; \boldsymbol{k}\right), \\
& h_{g}\left(\eta_{a}, \eta_{b}, y_{c}, \zeta_{B}, \boldsymbol{q}, \mu\right) \\
& \quad=\int \frac{d^{2} \boldsymbol{k}}{\pi} C_{g g^{*}}^{h}\left(\zeta_{B}, y_{c}, \eta_{a}, \boldsymbol{q}, \boldsymbol{k}, \mu\right) \mathcal{G}\left(\Delta \eta_{a b}, \eta_{b} ; \boldsymbol{k}\right) .
\end{aligned}
$$

Note that the TMD PDFs at first do not depend on the proton momentum fraction $x$, since high energy factorization requires to integrate over this longitudinal momentum fraction. Such a dependence therefore only arises through a special choice for the parameters $\eta_{a, b}$. To allow for a separate discussion of the different contributions of the gluon TMD, we further define
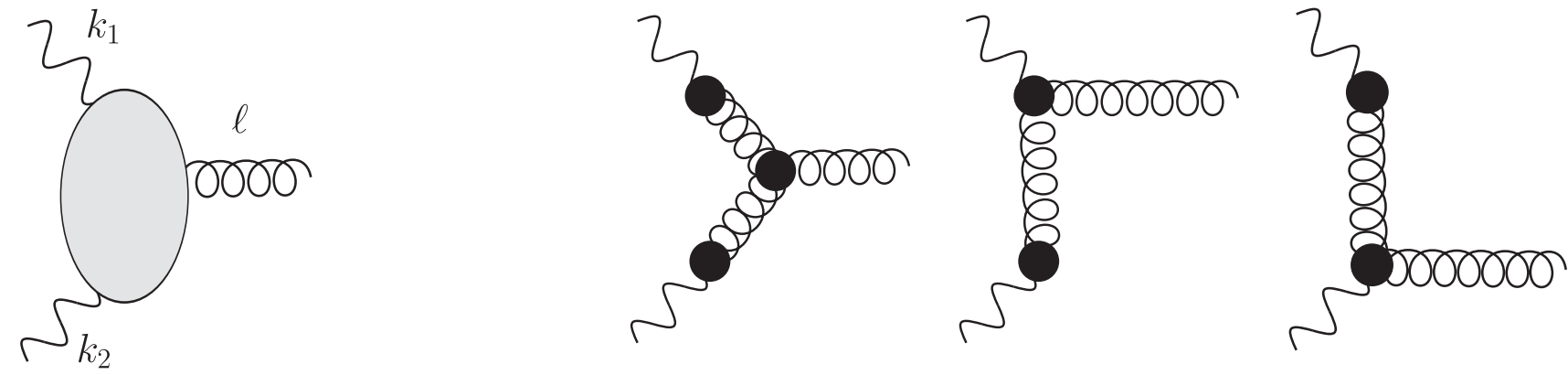

FIG. 3. Real emission contribution to the Lipatov vertex, which yields the one-loop Green's function (left) and contributing Feynman diagrams (right). Note current conservation of the Lipatov vertex is given both for tilted and untilted light cone directions. 


$$
\begin{aligned}
J^{i j}\left(q^{-}, \sigma, \boldsymbol{q}, \mu\right) & =\left.\int \frac{d \xi^{+} d^{2+2 \epsilon} \boldsymbol{\xi}}{2(2 \pi)^{3+2 \epsilon} p_{B}^{-}} e^{i q \cdot \xi}\left\langle h\left(p_{B}\right)\left|\operatorname{tr}\left[\left(\mathcal{W}_{\xi}^{n(\sigma)} G^{-i}(\xi)\right)^{\dagger} \mathcal{W}_{0}^{n(\sigma)} G^{-j}(0)\right]\right| h\left(p_{B}\right)\right\rangle\right|_{\xi^{-}=0} \\
\mathcal{S}\left(y_{c}, \sigma, \boldsymbol{q}, \mu\right) & =\int \frac{d^{2+2 \epsilon} \boldsymbol{\xi}}{(2 \pi)^{2}} e^{i \boldsymbol{q}_{2} \cdot \boldsymbol{\xi}} \tilde{\mathcal{S}}\left(y_{c}, \sigma, \boldsymbol{\xi}, \mu\right),
\end{aligned}
$$

where for the moment, we define the TMD PDF in $2+2 \epsilon$ dimensions, since individual expressions are divergent and $q^{-}=x p_{B}^{-}$. We therefore obtain

$$
x \Gamma^{i j}\left(q^{-}, y_{c}, \boldsymbol{q}, \mu\right)=\int d^{2+2 \epsilon} \boldsymbol{q}_{1} J^{i j}\left(q^{-}, \sigma, \boldsymbol{q}_{1}, \mu\right) \cdot \mathcal{S}\left(\left(y_{c}, \sigma, \boldsymbol{q}-\boldsymbol{q}_{1}, \mu\right) .\right.
$$

In the following, we evaluate the above gluon TMD for an initial reggeized gluon state with polarization $n^{+}$at one-loop. To be precise, we consider

$$
\bar{J}^{i j}\left(q^{-}, \sigma, \boldsymbol{q}, \boldsymbol{k}, \mu\right)=\left.\int \frac{d \xi^{+} d^{2+2 \epsilon} \boldsymbol{\xi}}{2(2 \pi)^{3+2 \epsilon} k^{-}} e^{i q \cdot \xi} \cdot \frac{1}{N_{c}^{2}-1} \sum_{b, b^{\prime}}\left\langle r_{+}^{b^{\prime}}(k)\left|\operatorname{tr}\left[\left(\mathcal{W}_{\xi}^{n(\sigma)} G^{-i}(\xi)\right)^{\dagger} \mathcal{W}_{0}^{n(\sigma)} G^{-j}(0)\right]\right| r_{+}^{b}(k)\right\rangle\right|_{\xi^{-}=0},
$$

where the reggeized gluon state $r^{b}(k)$ is defined with the normalization Eq. (17), appropriate for matching to collinear factorization in the limit of vanishing transverse momentum, i.e.,

$$
v_{\mu}^{a}(\xi)\left|r_{+}^{b}(k)\right\rangle=\left.e^{i k \cdot \xi} n_{\mu}^{+} \delta^{a b} \frac{k^{-}}{2|\boldsymbol{k}|}\right|_{k^{+}=0},
$$

while high energy factorization requires to integrate over the minus momentum,

$$
\hat{J}^{i j}\left(x, \boldsymbol{q}_{1}, \boldsymbol{k}\right)=\int \frac{d k^{-}}{k^{-}} \bar{J}^{i j}\left(x, \boldsymbol{q}_{1}, \boldsymbol{k}\right) .
$$

Feynman rules for the determination of perturbative corrections are summarized in Fig. 4. With the following convention to denote the perturbative expansion in $\alpha_{s}$ for a generic quantity $A\left(\alpha_{s}\right)$,

$$
A\left(\alpha_{s}\right)=A^{(0)}+A^{(1)}\left(\alpha_{s}\right)+\cdots,
$$
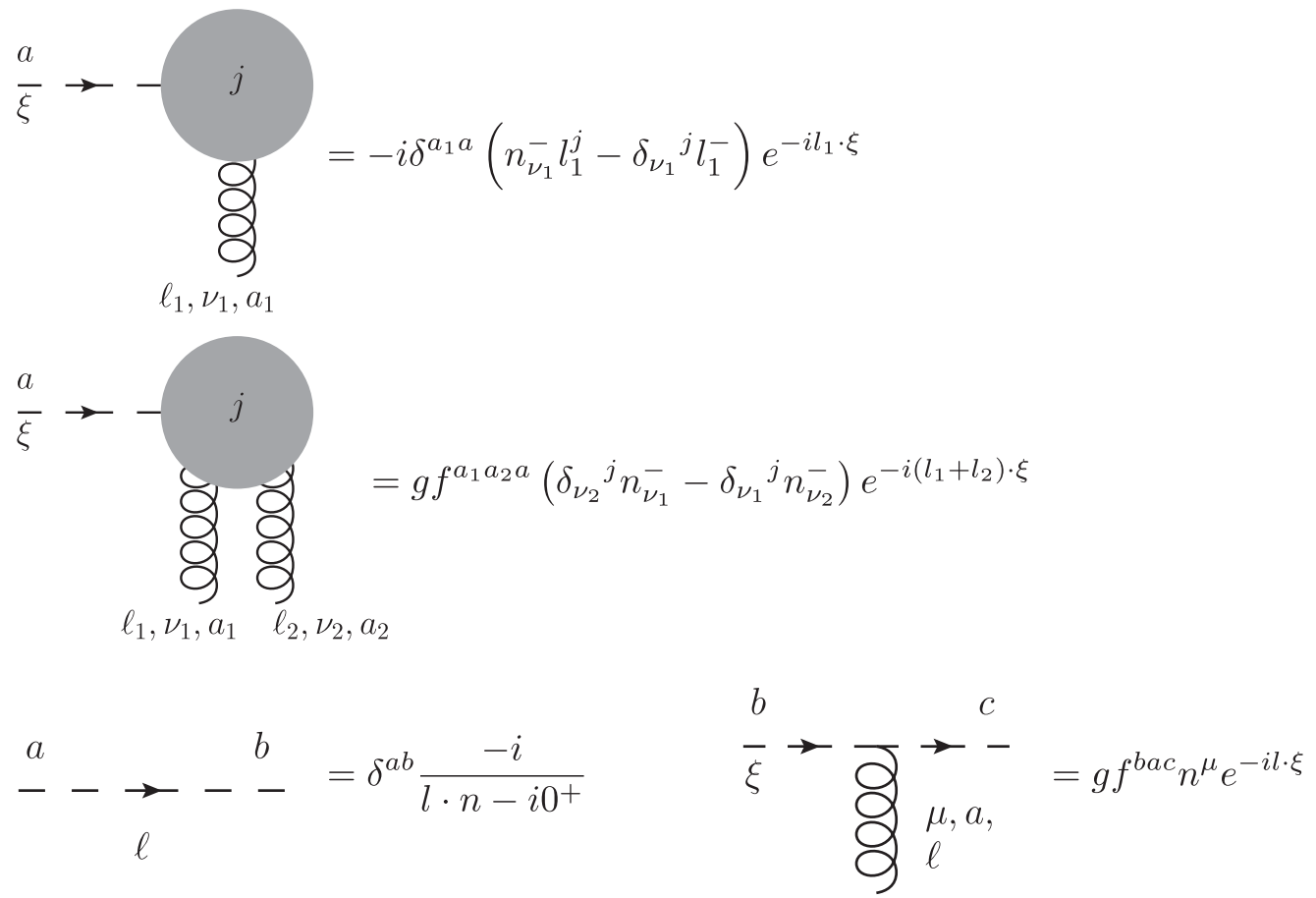

FIG. 4. Feynman rules for the perturbative determination of the gluon TMD at amplitude level, i.e., for the evaluation of $\left.\mathcal{W}_{0}^{n(\sigma)} G^{-j}(0)\right]\left|r_{+}^{b}(k)\right\rangle$. 
where $A^{(n)} \sim \alpha_{s}^{n}$, we have finally at leading order,

$$
\begin{aligned}
& \overline{\boldsymbol{J}}^{i j,(0)}=\frac{\boldsymbol{q}^{i} \boldsymbol{q}^{j}}{\boldsymbol{q}^{2}} \delta^{(2+2 \epsilon)}(\boldsymbol{q}-\boldsymbol{k}) \delta\left(1-\frac{k^{-}}{q^{-}}\right), \\
& \hat{\boldsymbol{J}}^{i j,(0)}=\frac{\boldsymbol{q}^{i} \boldsymbol{q}^{j}}{\boldsymbol{q}^{2}} \delta^{(2+2 \epsilon)}(\boldsymbol{q}-\boldsymbol{k}),
\end{aligned}
$$

and therefore,

$$
C_{g g^{*}}^{f,(0)}(\boldsymbol{q}, \boldsymbol{k})=C_{g g^{*}}^{h,(0)}(\boldsymbol{q}, \boldsymbol{k})=\delta^{(2)}(\boldsymbol{q}-\boldsymbol{k}),
$$

and the TMD gluon distributions are up to an overall factor [82] of $1 / \pi$ at leading order identical to the unintegrated gluon density [17],

$$
\begin{aligned}
f^{(0)} & =h^{(0)}=\frac{1}{\pi} G\left(\Delta \eta_{a b}, \eta_{b}, \boldsymbol{q}, \bar{f}^{(1)}\right), \\
\Gamma^{(0) i j} & =\frac{\boldsymbol{q}^{i} \boldsymbol{q}^{j}}{\boldsymbol{q}^{2} \pi} G\left(\Delta \eta_{a b}, \eta_{b}, \boldsymbol{q}, \bar{f}^{(1)}\right),
\end{aligned}
$$

where the $k_{T}$-factorization scheme defined in Sec. III C yields expression which are closest to conventional collin- ear factorization results. Note that the unintegrated gluon density is therefore directly related to the operator definition of the gluon TMD. Moreover, in the dilute limit, i.e., considering only two reggeized gluon exchange, the unintegrated gluon density is universal [83]. We further stress that the distribution of linearly polarized gluons in an unpolarized hadron is nonzero within high energy factorization already at tree level, in contrast to the result found within collinear factorization [64]. From a technical point of view, this is of course easily understood, since the initial gluon carries within high energy factorization already finite $\boldsymbol{k}$ and therefore gives rise to such a distribution.

\section{A. One-loop calculation without soft factor}

To regularize infrared and ultraviolet divergences, we use dimensional regularization in $d=4+2 \epsilon$. The virtual oneloop correction is provided by the set of diagrams Fig. 5 . The last diagram in the second line vanish within dimensional regularization, since it is scaleless. Including the contribution from the complex conjugate amplitude, we obtain the following result:

$$
\begin{aligned}
\bar{J}_{\text {virt }}^{i j,(1)}\left(q^{-}, \boldsymbol{q} ; k^{-}, \boldsymbol{k}\right) & =\delta\left(1-\frac{k^{-}}{q^{-}}\right) \hat{J}_{\mathrm{virt}}^{i j,(1)}\left(q^{-}, \boldsymbol{q} ; \boldsymbol{k}\right), \hat{J}_{\mathrm{virt}}^{i j,(1)}\left(q^{-}, \boldsymbol{q} ; \boldsymbol{k}\right) \\
& =\frac{\boldsymbol{q}^{i} \boldsymbol{q}^{j}}{\boldsymbol{q}^{2}} \delta^{(2+2 \epsilon)}(\boldsymbol{q}-\boldsymbol{k})\left(\frac{\boldsymbol{q}^{2}}{\mu^{2}}\right)^{\epsilon} \cdot \frac{\alpha_{s} C_{A}}{2 \pi}\left[\frac{1}{\epsilon^{2}}+\frac{1}{\epsilon}\left(-\rho+\ln \frac{q^{-2}}{\boldsymbol{q}^{2}}-\frac{8}{3}+\frac{2 n_{f}}{3 C_{A}}\right)+\frac{49}{9}-\frac{10 n_{f}}{9 C_{A}}-\frac{\pi^{2}}{3}\right]+\mathcal{O}(\epsilon),
\end{aligned}
$$

To obtain the projections on the distribution for the unpolarized TMD PDF $f^{g}$ and the linearly polarized gluons $h^{g}$ in an unpolarized hadron, we use

$$
\begin{aligned}
& f_{g / B}(x, \boldsymbol{q})=-g_{\perp, i j} \Gamma_{g / B}^{i j}(x, \boldsymbol{q}), \\
& h_{g / B}(x, \boldsymbol{q})=\frac{2+2 \epsilon}{1+2 \epsilon}\left(\frac{g^{i j}}{2+2 \epsilon}+\frac{\boldsymbol{q}^{i} \boldsymbol{q}^{j}}{\boldsymbol{q}^{2}}\right) \Gamma_{g / B}^{i j}(x, \boldsymbol{q}),
\end{aligned}
$$

which amounts to replace the overall tensor structure $\boldsymbol{q}^{i} \boldsymbol{q}^{j} / \boldsymbol{q}^{2}$ by one for both the unpolarized and the linearly polarized TMD. In particular, due to the presence of nonzero initial transverse momentum, the virtual correction for both TMD distributions are nonzero and agree with each other. Diagrams for real corrections are depicted in Fig. 6. Parametrizing $k^{-}=q^{-} / z$ and correspondingly, $l^{-}=q^{-}(1-z) / z$, a straightforward calculation yields

$$
\begin{aligned}
\bar{J}_{\text {real }}^{i j,(1)}\left(q^{-}, \boldsymbol{q} ; k^{-}, \boldsymbol{k}\right)= & \frac{1}{\pi^{1+\epsilon}} \int_{0}^{1} d z \delta\left(1-\frac{z k^{-}}{q^{-}}\right) \\
& \times \frac{1}{(\boldsymbol{q}-\boldsymbol{k})^{2}} \tilde{P}_{g g, r}^{(0), i j}(z, \boldsymbol{q}, \boldsymbol{k}),
\end{aligned}
$$

where
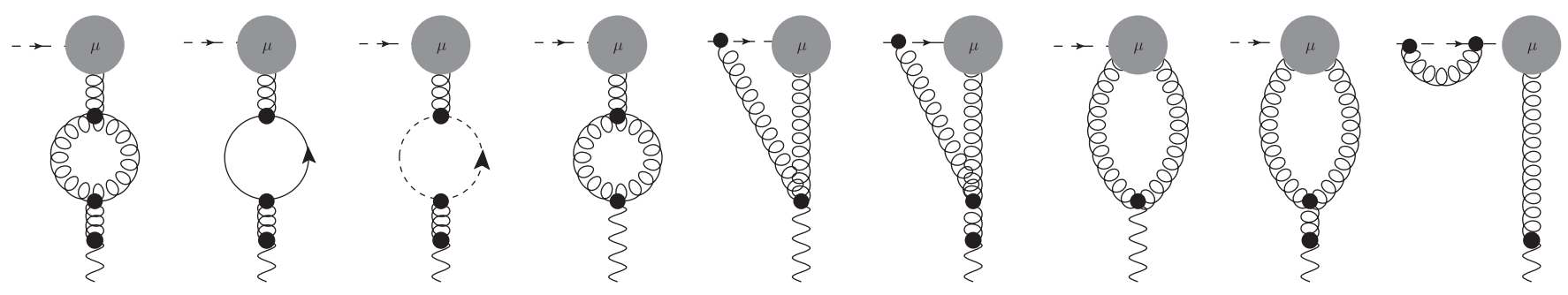

FIG. 5. Virtual corrections. 

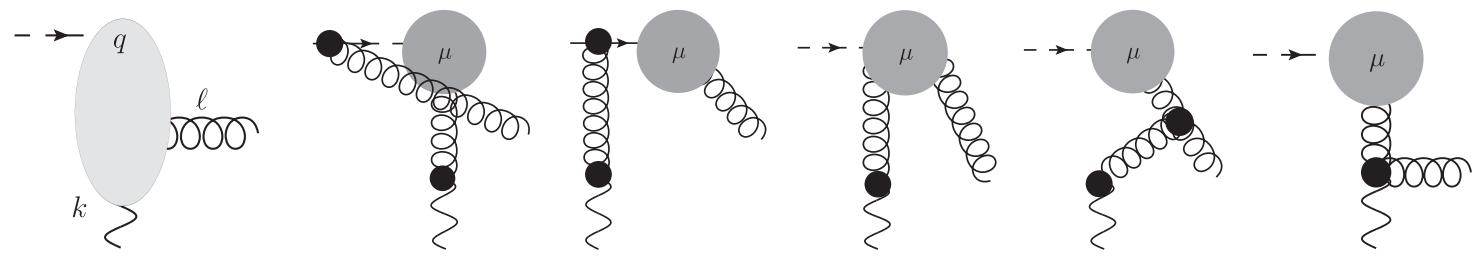

FIG. 6. Real corrections. Left: The reggeized gluon $(k)$ is taken as incoming, the real gluon $(\ell)$ and the momentum of the TMD PDF $(q)$ as outgoing, $k=q+\ell$. Right: Contributing Feynman diagrams.

$$
\begin{aligned}
\frac{1}{(\boldsymbol{q}-\boldsymbol{k})^{2}} P_{g g, r}^{(0) i j}(z, \boldsymbol{q}, \boldsymbol{k})= & \frac{\alpha_{s} C_{A}}{2 \pi \mu^{2 \epsilon} \Gamma(1-\epsilon)}\left[\frac{-g_{\perp}^{i j}}{2} \frac{z(1-z)\left((\boldsymbol{q}-\boldsymbol{k})^{2}-\boldsymbol{q}^{2}\right)^{2}}{\left(z(\boldsymbol{q}-\boldsymbol{k})^{2}+(1-z) \boldsymbol{q}^{2}\right) \boldsymbol{k}^{2}}+\frac{\boldsymbol{q}^{i} \boldsymbol{q}^{j}}{\boldsymbol{q}^{2}}\left(\frac{2}{z\left[(\boldsymbol{q}-\boldsymbol{k})^{2}+e^{-\rho} \frac{(1-z)^{2}}{z^{2}} q^{-2}\right]}\right.\right. \\
& \left.-\frac{2}{z(\boldsymbol{q}-\boldsymbol{k})^{2}+(1-z) \boldsymbol{q}^{2}}\right)+\frac{\boldsymbol{k}^{\boldsymbol{i}} \boldsymbol{k}^{j}}{\boldsymbol{k}^{2}(\boldsymbol{q}-\boldsymbol{k})^{2}}\left(\frac{2 \boldsymbol{q}^{2}}{\left[(1-z)+e^{-\sigma} \frac{(\boldsymbol{q}-\boldsymbol{k})^{2} z^{2}}{(1-z)^{2} q^{-2}}\right]}-\frac{1}{\left[z(\boldsymbol{q}-\boldsymbol{k})^{2}+(1-z) \boldsymbol{q}^{2}\right]}\right) \\
& \left.+\frac{\boldsymbol{k}^{i} \boldsymbol{q}^{j}+\boldsymbol{q}^{i} \boldsymbol{k}^{j}}{(\boldsymbol{q}-\boldsymbol{k})^{2}} \frac{1}{\left[z(\boldsymbol{q}-\boldsymbol{k})^{2}+(1-z) \boldsymbol{q}^{2}\right]}\right]+\mathcal{O}\left(e^{-\rho}, e^{-\sigma}\right),
\end{aligned}
$$

where we kept only track of those contributions of order $e^{-\rho}$ and $e^{-\sigma}$, which are needed to regulate integrals over the momentum fraction $z$ and/or transverse momenta, which are not convergent within dimensional regularization. Real splitting functions for the unpolarized $\left(\tilde{P}_{g g}^{(f)}\right)$ and linearly polarized $\left(\tilde{P}_{g g}^{(h)}\right)$ gluon read

$$
\begin{aligned}
& \frac{1}{\boldsymbol{l}^{2}} \tilde{P}_{g g, r}^{(0) f}(z, \boldsymbol{q}, \boldsymbol{k})=\frac{\alpha_{s} C_{A}}{2 \pi \mu^{2 \epsilon} \Gamma(1-\epsilon)}\left\{\frac{z(1-z)(1+\epsilon)\left(\boldsymbol{l}^{2}-\boldsymbol{q}^{2}\right)^{2}}{\left[z \boldsymbol{l}^{2}+(1-z) \boldsymbol{q}^{2}\right]^{2} \boldsymbol{k}^{2}}+\frac{2}{\boldsymbol{l}^{2}}\left[\frac{1}{z \cdot\left[1+e^{-\rho} \frac{(1-z)^{2} q^{-2}}{z^{2} \boldsymbol{l}^{2}}\right]}+\frac{1}{(1-z) \cdot\left[1+e^{-\sigma} \frac{\boldsymbol{l}^{2} z^{2}}{(1-z)^{2} q^{-2}}\right]}\right]\right. \\
& \left.+\frac{1}{\boldsymbol{l}^{2}} \frac{\boldsymbol{k}^{2}-3(\boldsymbol{q}-\boldsymbol{k})^{2}-\boldsymbol{q}^{2}}{\left[z(\boldsymbol{q}-\boldsymbol{k})^{2}+(1-z) \boldsymbol{q}^{2}\right]}\right\} \\
& \frac{1}{\boldsymbol{l}^{2}} \tilde{P}_{g g, r}^{(0) h}(z, \boldsymbol{q}, \boldsymbol{k})=\frac{\alpha_{s} C_{A}}{2 \pi \mu^{2 \epsilon} \Gamma(1-\epsilon)}\left\{\frac{2}{z \boldsymbol{l}^{2} \cdot\left[1+e^{-\rho} \frac{(1-z)^{2} q^{-2}}{z^{2} \boldsymbol{l}^{2}}\right]}+\frac{2}{(1-z) \boldsymbol{l}^{2} \cdot\left[1+e^{-\sigma} \frac{\boldsymbol{l}^{2} z^{2}}{(1-z)^{2} q^{-2}}\right]}\left(1+\frac{4(1+\epsilon)\left((\boldsymbol{l} \cdot \boldsymbol{q})^{2}-\boldsymbol{l}^{2} \boldsymbol{q}^{2}\right)}{(1+2 \epsilon) \boldsymbol{l}^{2} \boldsymbol{q}^{2} \boldsymbol{k}^{2}}\right)\right. \\
& \left.+\frac{1}{\left[z \boldsymbol{l}^{2}+(1-z) \boldsymbol{q}^{2}\right]}\left(\frac{(1+2 \epsilon) 2 \boldsymbol{k} \cdot \boldsymbol{q}+2 \boldsymbol{q}^{2}}{\boldsymbol{l}^{2}(1+2 \epsilon)}-\frac{2(1+\epsilon)(\boldsymbol{k} \cdot \boldsymbol{q})^{2}}{\boldsymbol{k}^{2} \boldsymbol{l}^{2}(1+2 \epsilon)}-2\right)\right\},
\end{aligned}
$$

where we used $\boldsymbol{l}=\boldsymbol{k} \boldsymbol{- \boldsymbol { q }}$. Note that the splitting function corresponding to $f_{g}$ coincides with the real transverse momentum splitting function derived in [34] if we set $\rho=\infty=\sigma$. As demonstrated in [34], this splitting function coincides with the DGLAP splitting function in the limit $\boldsymbol{k} \rightarrow 0$, reduces to the real part of the leading order BFKL kernel in the limit $z \rightarrow 0$, and yields the real leading order kernel of the CCFM equation in the limit $\boldsymbol{q} \rightarrow \boldsymbol{k}$. The present calculation provides on the other hand an opportunity to determine the still missing virtual contribution to this splitting function. We further note-in agreement with the result presented in [64] - that the coefficient of the singularity $z \rightarrow 1$ vanishes for the polarized splitting,
Eq. (64) in the collinear limit $\boldsymbol{k} \rightarrow 0$, after averaging additionally over the azimuthal angle of the incoming gluon. For finite $\boldsymbol{k}$, this singularity is on the other hand present and requires a treatment similar to the case of the unpolarized gluon TMD PDF. With the integrated real corrections-relevant for the current discussion which is based on high energy factorization-we finally have

$$
\hat{J}_{\text {real }}^{i j,(1)}\left(q^{-}, \boldsymbol{q} ; \boldsymbol{k}\right)=\int_{0}^{1} d z \frac{1}{(\boldsymbol{q}-\boldsymbol{k})^{2}} \frac{\tilde{P}_{g g, r}^{(0), i j}(z, \boldsymbol{q}, \boldsymbol{k})}{\pi^{1+\epsilon}},
$$

with

$$
\hat{J}_{\text {real }}^{(1) f}\left(q^{-}, \boldsymbol{q} ; \boldsymbol{k}\right)=\frac{\alpha_{s} C_{A}}{2 \pi \pi_{\epsilon}}\left\{\frac{\sigma+\rho}{\boldsymbol{l}^{2}}+\int_{0}^{1} d z\left[\frac{z(1-z)(1+\epsilon)\left(\boldsymbol{l}^{2}-\boldsymbol{q}^{2}\right)^{2}}{\left[z \boldsymbol{l}^{2}+(1-z) \boldsymbol{q}^{2}\right]^{2} \boldsymbol{k}^{2}}+\frac{1}{\boldsymbol{l}^{2}} \frac{\boldsymbol{k}^{2}-3(\boldsymbol{q}-\boldsymbol{k})^{2}-\boldsymbol{q}^{2}}{\left[z(\boldsymbol{q}-\boldsymbol{k})^{2}+(1-z) \boldsymbol{q}^{2}\right]}\right]\right\},
$$




$$
\begin{aligned}
\hat{J}_{\text {real }}^{(1) h}\left(q^{-}, \boldsymbol{q} ; \boldsymbol{k}\right)= & \frac{\alpha_{s} C_{A}}{2 \pi \pi_{\epsilon}}\left\{\frac{\sigma+\rho}{\boldsymbol{l}^{2}}+\left(\sigma+\ln \frac{q^{-2}}{\boldsymbol{l}^{2}}\right) \frac{2(1+\epsilon)\left((\boldsymbol{l} \cdot \boldsymbol{q})^{2}-\boldsymbol{l}^{2} \boldsymbol{q}^{2}\right)}{(1+2 \epsilon) \boldsymbol{l}^{2} \boldsymbol{q}^{2} \boldsymbol{k}^{2}}\right. \\
& \left.+\int_{0}^{1} d z \frac{1}{\left[z \boldsymbol{l}^{2}+(1-z) \boldsymbol{q}^{2}\right]}\left(\frac{(1+2 \epsilon) 2 \boldsymbol{k} \cdot \boldsymbol{q}+2 \boldsymbol{q}^{2}}{\boldsymbol{l}^{2}(1+2 \epsilon)}-\frac{4(1+\epsilon)(\boldsymbol{k} \cdot \boldsymbol{q})^{2}}{\boldsymbol{k}^{2} \boldsymbol{l}^{2}(1+2 \epsilon)}-2\right)\right\},
\end{aligned}
$$

where the remaining integrals over $z$ are finite and $\pi_{\epsilon}$ is defined in Eq. (32). If inserted into Eq. (49), the convolution integral over the reggeized gluon momentum $\boldsymbol{k}$ gives still rise to an infrared singularity. A possibility to extract these singularities is the use of a phase space slicing parameter; see, e.g., [84-86] for an example within the high energy effective action. While this is sufficient to demonstrate finiteness at a formal level, the use of such phase space slicing parameters is in general complicated for numerical studies at NLO accuracy. For the case of collinear NLO calculation, the by now conventional tool to overcome this difficulty are subtraction methods, in particular dipole subtraction [87]. In [62], this has been slightly generalized and applied to the case of divergences, which arise due to convolution integrals of transverse momenta. In particular, the following decomposition has been proposed:

$$
\begin{aligned}
\int \frac{d^{2+2 \epsilon}}{\pi^{1+\epsilon}} \frac{\boldsymbol{l}}{\boldsymbol{l}^{2}} G((\boldsymbol{l}) & \int \frac{d^{2} \boldsymbol{l}}{\pi}\left[\frac{\kappa(\boldsymbol{l})}{\boldsymbol{l}^{2}}\right]_{+} G\left((\boldsymbol{q}+\boldsymbol{l})^{2}\right) \\
& +\int \frac{d^{2+2 \varepsilon} \boldsymbol{l}}{\pi^{1+\varepsilon}} \frac{\kappa(\boldsymbol{l})}{\boldsymbol{l}^{2}} \frac{\boldsymbol{q}^{2} G\left(\boldsymbol{q}^{2}\right)}{\boldsymbol{l}^{2}+(\boldsymbol{q}+\boldsymbol{l})^{2}}+\mathcal{O}(\epsilon),
\end{aligned}
$$

with

$$
\int \frac{d^{2} \boldsymbol{l}}{\pi}\left[\frac{\kappa(\boldsymbol{l})}{\boldsymbol{l}^{2}}\right]_{+} G\left((\boldsymbol{q}+\boldsymbol{l})^{2}\right) \equiv \int \frac{d^{2} \boldsymbol{l}}{\pi} \frac{\kappa(\boldsymbol{l})}{\boldsymbol{l}^{2}}\left[G\left((\boldsymbol{q}+\boldsymbol{l})^{2}\right)-\frac{\boldsymbol{q}^{2} G\left(\boldsymbol{q}^{2}\right)}{\boldsymbol{l}^{2}+(\boldsymbol{q}+\boldsymbol{l})^{2}}\right]
$$

The expression in the squared brackets on the right-hand side vanish in the limit $|\boldsymbol{l}| \rightarrow 0$, and $G(\boldsymbol{k})$ is a function which parametrizes the transverse momentum dependence of the reggeized gluon state. The function $\kappa(l)$ is such that the integral on the right-hand side of (69) is well-defined, which in practice means that it does not behave worse than $\ln |\boldsymbol{l}|$ for $|\boldsymbol{l}| \rightarrow 0$ and $|\boldsymbol{l}| \rightarrow \infty$. Furthermore, it should be such that the integral in the second line of (68) can be calculated analytically. Note that the factor $\boldsymbol{q}^{2} /\left[\boldsymbol{l}^{2}+(\boldsymbol{q}+\boldsymbol{l})^{2}\right]$ is needed to achieve convergence in the ultraviolet. In the current setup, we merely require the case $\kappa(\boldsymbol{l})=1$ with

$$
\int \frac{d^{2+2 \epsilon} \boldsymbol{l}}{\pi^{1+\epsilon}} \frac{1}{\boldsymbol{l}^{2}\left[\boldsymbol{l}^{2}+(\boldsymbol{q}+\boldsymbol{l})^{2}\right]}=\frac{\Gamma(1-\epsilon)}{\epsilon\left(\boldsymbol{q}^{2}\right)^{1-\epsilon}}+\mathcal{O}(\epsilon)
$$

and

$$
\begin{aligned}
\hat{J}_{\text {real }}^{(1) f}\left(q^{-}, \boldsymbol{q} ; \boldsymbol{k}\right)= & \frac{\alpha_{s} C_{A}}{2 \pi}\left\{(\rho+\sigma)\left(\delta^{(2)}(\boldsymbol{l}) \frac{1}{\epsilon}\left(\frac{\boldsymbol{q}^{2}}{\mu^{2}}\right)^{\epsilon}+\frac{1}{\pi^{1+\epsilon}}\left[\frac{1}{\boldsymbol{l}^{2}}\right]_{+}\right)\right. \\
& \left.+\frac{1}{\pi^{1+\epsilon}} \int_{0}^{1} d z\left[\frac{z(1-z)\left(\boldsymbol{l}^{2}-\boldsymbol{q}^{2}\right)^{2}}{\left[z \boldsymbol{l}^{2}+(1-z) \boldsymbol{q}^{2}\right]^{2} \boldsymbol{k}^{2}}+\frac{1}{\boldsymbol{l}^{2}} \frac{\boldsymbol{k}^{2}-3(\boldsymbol{q}-\boldsymbol{k})^{2}-\boldsymbol{q}^{2}}{\left[z(\boldsymbol{q}-\boldsymbol{k})^{2}+(1-z) \boldsymbol{q}^{2}\right]}\right]\right\}+\mathcal{O}(\epsilon), \\
\hat{J}_{\text {real }}^{(1) h}\left(q^{-}, \boldsymbol{q} ; \boldsymbol{k}\right)= & \frac{\alpha_{s} C_{A}}{2 \pi}\left\{(\rho+\sigma)\left(\delta^{(2)}(\boldsymbol{l}) \frac{1}{\epsilon}\left(\frac{\boldsymbol{q}^{2}}{\mu^{2}}\right)^{\epsilon}+\frac{1}{\pi^{1+\epsilon}}\left[\frac{1}{\boldsymbol{l}^{2}}\right]_{+}\right)\right. \\
& \left.+\left(\sigma+\ln \frac{q^{-2}}{\boldsymbol{l}^{2}}\right) \frac{2\left((\boldsymbol{l} \cdot \boldsymbol{q})^{2}-\boldsymbol{l}^{2} \boldsymbol{q}^{2}\right)}{\pi^{1+\epsilon} \boldsymbol{l}^{2} \boldsymbol{q}^{2} \boldsymbol{k}^{2}}+\frac{1}{\pi^{1+\epsilon}} \int_{0}^{1} d z \frac{\left(\boldsymbol{q}^{2}-\boldsymbol{l}^{2}\right) 2 \boldsymbol{k} \cdot \boldsymbol{l}}{\left[z \boldsymbol{l}^{2}+(1-z) \boldsymbol{q}^{2}\right] \boldsymbol{l}^{2} \boldsymbol{k}^{2}}\right\}+\mathcal{O}(\epsilon),
\end{aligned}
$$

where the corresponding convolution integral can now be defined in $d=2$ dimensions. 


\section{B. Soft factor, counterterms, and renormalization}

The above result contains both rapidity divergences due to high energy factorization $(\rho \rightarrow \infty)$ and the TMD definition $(\sigma \rightarrow \infty)$ as well as single and double poles in $1 / \epsilon$. Rapidity divergences due to high energy factorization require the subtraction of high energy factorized contributions to the above correlator as well as the application of the transition function, as given by Eq. (37). Rapidity divergences due to soft radiation require the soft factor Eq. (10), which we did not include so far. With the one-loop expansion,

$$
\begin{aligned}
S\left(y_{1}, y_{2} ; \boldsymbol{q}_{2}\right)= & \delta^{(2+2 \epsilon)}\left(\boldsymbol{q}_{2}\right) \\
& +\frac{\alpha_{s} C_{A}}{2 \pi \pi_{\epsilon}}\left(y_{1}+y_{2}\right) \frac{1+e^{-y_{1}-y_{2}}}{1-e^{-y_{1}-y_{2}}} \frac{1}{\boldsymbol{q}_{2}^{2}}+\mathcal{O}\left(\alpha_{s}^{2}\right),
\end{aligned}
$$

and taking the limit [88] $\sigma, y_{n} \rightarrow \infty$, we obtain finally for the soft function in momentum space at one loop,

$$
\mathcal{S}\left(2 y_{c}, \sigma ; \boldsymbol{q}_{2}\right)=\delta^{(2+2 \epsilon)}\left(\boldsymbol{q}_{2}\right)-\frac{\alpha_{s} C_{A}\left(\sigma-2 y_{c}\right)}{2 \pi \pi_{\epsilon} \boldsymbol{q}_{2}^{2}}+\mathcal{O}\left(\alpha_{s}^{2}\right),
$$

where $y_{c}$ is a finite rapidity and takes a role related to a factorization scale, similar to the parameter $\eta_{a}$ in the case of high energy factorization; in particular, it enters directly the definition of the scales $\zeta_{A, B}$ defined in Eq. (2). The oneloop contribution to the TMD gluon densities due to the soft function is finally given by

$$
\hat{\Gamma}_{\text {soft }}^{i j(1)}(x, \boldsymbol{q}, \boldsymbol{k})=-\frac{\alpha_{s} C_{A}\left(\sigma-2 y_{c}\right)}{2 \pi \pi_{\epsilon}(\boldsymbol{q}-\boldsymbol{k})^{2}} \frac{\boldsymbol{k}^{i} \boldsymbol{k}^{j}}{\boldsymbol{k}^{2}}+\mathcal{O}\left(\alpha_{s}^{2}\right) .
$$

Note that the one-loop soft function, which consists of its real part only within dimensional regularization, agrees with the real part of the one-loop BFKL kernel. We believe that this coincidence is limited to the one-loop case and does not hint at a general universality of the rapidity dependence of the soft function. In particular, at the diagrammatic level the soft function does not give rise to the complete Lipatov vertex, but only to terms related to the induced contributions; see also the discussion in [89] in the context of soft-collinear effective theory for high energy scattering.

As a last step, we need to combine virtual [Eq. (59) and real (Eqs. (66) (67)] corrections, with the soft factor, making use of the appropriate projections. Note that the contribution of the soft factor merely amounts to a replacement of the regulator $\sigma$ by the factorization parameter $y_{c}$. We obtain for the one-loop high energy subtracted and renormalized one-loop coefficients $\tilde{C}^{(1) f, h}$ the following result:

$$
\begin{aligned}
\tilde{C}^{(1) f}\left(q^{-}, \boldsymbol{q} ; \boldsymbol{k}\right)= & \frac{\alpha_{s} C_{A}}{2 \pi}\left\{\delta ^ { ( 2 ) } ( \boldsymbol { l } ) \left[\frac{1}{\epsilon^{2}}+\frac{1}{\epsilon}\left(\ln \frac{\left(q^{-}\right)^{2} e^{2 y_{c}}}{\mu^{2}}-\frac{\beta_{0}}{2 C_{A}}\right)+\frac{67}{18}-\frac{5}{9}-\frac{\pi^{2}}{3}+\ln \frac{\boldsymbol{q}^{2}}{\mu^{2}}\left(\ln \frac{\left(q^{-}\right)^{2} e^{2 y_{c}}}{\mu^{2}}-\frac{\beta_{0}}{2 C_{A}}\right)\right.\right. \\
& \left.-\frac{1}{2} \ln ^{2} \frac{\boldsymbol{q}^{2}}{\mu^{2}}\right]+2\left(y_{c}-\eta_{a}\right) \frac{1}{\pi^{1+\epsilon}}\left[\frac{1}{\boldsymbol{l}^{2}}\right]_{+}+\frac{1}{\pi^{1+\epsilon}} \int_{0}^{1} d z\left[\frac{z(1-z)\left(\boldsymbol{l}^{2}-\boldsymbol{q}^{2}\right)^{2}}{\left[z \boldsymbol{l}^{2}+(1-z) \boldsymbol{q}^{2}\right]^{2} \boldsymbol{k}^{2}}\right. \\
& \left.\left.+\frac{1}{\boldsymbol{l}^{2}} \frac{\boldsymbol{k}^{2}-3(\boldsymbol{q}-\boldsymbol{k})^{2}-\boldsymbol{q}^{2}}{\left[z(\boldsymbol{q}-\boldsymbol{k})^{2}+(1-z) \boldsymbol{q}^{2}\right]}\right]\right\}+\bar{f}^{(1)}(\boldsymbol{q}, \boldsymbol{k})+\mathcal{O}(\epsilon), \\
\tilde{\boldsymbol{C}}^{(1) h}\left(q^{-}, \boldsymbol{q} ; \boldsymbol{k}\right)= & \frac{\alpha_{s} C_{A}}{2 \pi}\left\{\delta ^ { ( 2 ) } ( \boldsymbol { l } ) \left[\frac{1}{\epsilon^{2}}+\frac{1}{\epsilon}\left(\ln \frac{\left(q^{-}\right)^{2} e^{2 y_{c}}}{\mu^{2}}-\frac{\beta_{0}}{2 C_{A}}\right)+\frac{67}{18}-\frac{5}{9}-\frac{\pi^{2}}{3}+\ln \frac{\boldsymbol{q}^{2}}{\mu^{2}}\left(\ln \frac{\left(q^{-}\right)^{2} e^{2 y_{c}}}{\mu^{2}}-\frac{\beta_{0}}{2 C_{A}}\right)\right.\right. \\
& \left.-\frac{1}{2} \ln ^{2} \frac{\boldsymbol{q}^{2}}{\mu^{2}}\right]+2\left(y_{c}-\eta_{a}\right) \frac{1}{\pi^{1+\epsilon}}\left[\frac{1}{\boldsymbol{l}^{2}}\right]+2\left(y_{c}+\ln \frac{q^{-}}{|\boldsymbol{l}|}\right) \frac{2\left((\boldsymbol{l} \cdot \boldsymbol{q})^{2}-\boldsymbol{l}^{2} \boldsymbol{q}^{2}\right)}{\pi^{1+\epsilon} \boldsymbol{l}^{2} \boldsymbol{q}^{2} \boldsymbol{k}^{2}} \\
& \left.+\frac{1}{\pi^{1+\epsilon}} \int_{0}^{1} d z \frac{\left(\boldsymbol{q}^{2}-\boldsymbol{l}^{2}\right) 2 \boldsymbol{k} \cdot \boldsymbol{l}}{\left[z \boldsymbol{l}^{2}+(1-z) \boldsymbol{q}^{2}\right] \boldsymbol{l}^{2} \boldsymbol{k}^{2}}\right\}+\bar{f}^{(1)}(\boldsymbol{q}, \boldsymbol{k})+\mathcal{O}(\epsilon),
\end{aligned}
$$

where

$$
\beta_{0}=\frac{11 C_{A}}{3}-\frac{2 n_{f}}{3} .
$$

While the above expression no longer carries rapidity divergences, it still comes with several poles in $1 / \epsilon$, which are of ultraviolet origin and which require renormalization.
The corresponding renormalization constant is identical for both unpolarized and linearly polarized gluons and is obtained as

$$
\mathcal{Z}_{G}=1-\frac{\alpha_{s} C_{A}}{2 \pi}\left[\frac{1}{\epsilon^{2}}+\frac{1}{\epsilon}\left(\ln \frac{\zeta}{\mu^{2}}-\frac{\beta_{0}}{2 C_{A}}\right)\right],
$$

which gives rise to the following anomalous dimension: 


$$
\begin{aligned}
\gamma_{G}\left(\alpha_{s}(\mu), \ln \frac{\zeta}{\mu^{2}}\right) & =\frac{d \ln \mathcal{Z}_{G}}{d \ln \mu} \\
& =\frac{\alpha_{S}}{2 \pi}\left[\beta_{0}-2 C_{A} \ln \frac{\zeta}{\mu^{2}}\right],
\end{aligned}
$$

where we used $d \alpha_{s} / d \ln \mu=2 \epsilon \alpha_{s}$ and $\zeta=\left(q^{-}\right)^{2} e^{2 y_{c}}$. Note that the above anomalous dimension agrees with the corresponding result obtained within a treatment based on collinear factorization [64]. This is indeed to be expected since it arises due to the renormalization of ultraviolet divergences, which are naturally independent of the nonzero transverse momentum of the initial state gluon. We however stress that the linearly polarized TMD gluon distribution does not give rise to the above anomalous dimension within collinear factorization, since the corresponding distribution vanishes within collinear factorization at tree level; the one-loop result is therefore not renormalized. We finally obtained for the renormalized coefficients,

$$
\begin{aligned}
\hat{C}_{g g *}^{(1) f}\left(\zeta_{B}, y_{c}, \eta_{a}, \boldsymbol{q}, \boldsymbol{k}, \mu, \bar{f}^{(1)}\right)= & \frac{\alpha_{s} C_{A}}{2 \pi}\left\{\delta^{(2)}(\boldsymbol{l})\left[\ln \frac{\boldsymbol{q}^{2}}{\mu^{2}}\left(\ln \frac{\zeta_{B}}{\mu^{2}}-\frac{\beta_{0}}{2 C_{A}}\right)-\frac{1}{2} \ln ^{2} \frac{\boldsymbol{q}^{2}}{\mu^{2}}+\frac{67}{18}-\frac{5 n_{f}}{9 C_{A}}-\frac{\pi^{2}}{3}\right]+2\left(y_{c}-\eta_{a}\right) \frac{1}{\pi}\left[\frac{1}{\boldsymbol{l}^{2}}\right]_{+}\right. \\
& \left.+\frac{1}{\pi} \int_{0}^{1} d z\left[\frac{z(1-z)\left(\boldsymbol{l}^{2}-\boldsymbol{q}^{2}\right)^{2}}{\left[z \boldsymbol{l}^{2}+(1-z) \boldsymbol{q}^{2}\right]^{2} \boldsymbol{k}^{2}}+\frac{1}{\boldsymbol{l}^{2}} \frac{\boldsymbol{k}^{2}-3(\boldsymbol{q}-\boldsymbol{k})^{2}-\boldsymbol{q}^{2}}{\left[z(\boldsymbol{q}-\boldsymbol{k})^{2}+(1-z) \boldsymbol{q}^{2}\right]}\right]\right\}+\bar{f}^{(1)}(\boldsymbol{q}, \boldsymbol{k}) \\
\hat{C}_{g g *}^{(1) h}\left(\zeta_{B}, y_{c}, \eta_{a}, \boldsymbol{q}, \boldsymbol{k}, \mu, \bar{f}^{(1)}\right)= & \frac{\alpha_{s} C_{A}}{2 \pi}\left\{\delta^{(2)}(\boldsymbol{l})\left[\ln \frac{\boldsymbol{q}^{2}}{\mu^{2}}\left[\ln \frac{\zeta_{B}}{\mu^{2}}-\frac{\beta_{0}}{2 C_{A}}\right]-\frac{1}{2} \ln ^{2} \frac{\boldsymbol{q}^{2}}{\mu^{2}}+\frac{67}{18}-\frac{5 n_{f}}{9 C_{A}}-\frac{\pi^{2}}{3}\right]+2\left(y_{c}-\eta_{a}\right) \frac{1}{\pi}\left[\frac{1}{\boldsymbol{l}^{2}}\right]_{+}\right. \\
& \left.+\left(y_{c}+\ln \frac{q^{-}}{|\boldsymbol{l}|}\right) \frac{4\left((\boldsymbol{l} \cdot \boldsymbol{q})^{2}-\boldsymbol{l}^{2} \boldsymbol{q}^{2}\right)}{\pi \boldsymbol{l}^{2} \boldsymbol{q}^{2} \boldsymbol{k}^{2}}+\frac{1}{\pi} \int_{0}^{1} d z \frac{\left(\boldsymbol{q}^{2}-\boldsymbol{l}^{2}\right) 2 \boldsymbol{k} \cdot \boldsymbol{l}}{\left[z \boldsymbol{l}^{2}+(1-z) \boldsymbol{q}^{2}\right] \boldsymbol{l}^{2} \boldsymbol{k}^{2}}\right\}+\bar{f}^{(1)}(\boldsymbol{q}, \boldsymbol{k}),
\end{aligned}
$$

where we made now the dependence on various parameters $y_{c}$ and $\eta_{a}$ explicit. The above expressions for the one-loop coefficients of unpolarized and linearly polarized gluon TMD are one of the main results of this work.

\section{EVOLUTION}

The coefficients Eqs. (81), (82) depend on three factorization scales and/or parameters: $\mu$ (renormalization scale), $y_{c}$ (evolution parameter of the soft function), and $\eta_{a}$ (evolution parameter of the unintegrated gluon density). In addition, we still have the dependence on the function $\bar{f}^{(1)}$, which is also related to the unintegrated gluon density. The dependence on the renormalization scale and the factorization parameter $y_{c}$ gives rise to CSS resummation framework, [4-6]. In the treatment established for collinear initial states, see, e.g., $[2,64,90]$, it is customary to consider to this end the Fourier-transform of the TMD coefficient to transverse coordinate space,

$$
\begin{aligned}
& \tilde{C}_{g g^{*}}^{(1) i}\left(\zeta_{B}, y_{c}, \eta_{a}, \boldsymbol{b}, \boldsymbol{k}, \mu, \bar{f}^{(1)}\right) \\
& \quad=\int d^{2} \boldsymbol{q} e^{i \boldsymbol{q} \cdot \boldsymbol{b}} \hat{\boldsymbol{C}}_{g g^{*}}^{(1) i}\left(\zeta_{B}, y_{c}, \eta_{a}, \boldsymbol{q}, \boldsymbol{k}, \mu, \bar{f}^{(1)}\right),
\end{aligned}
$$

where $i=f, h$ and then to evolve the coefficient in coordinate space,

$$
\begin{aligned}
\tilde{C}_{g g *}^{(1) i} & \left(\zeta_{B, f}, \ln \frac{\sqrt{\zeta_{B, f}}}{q^{-}}, \eta_{a}, \boldsymbol{b}, \boldsymbol{k}, \mu_{f}, \bar{f}^{(1)}\right) \\
= & \tilde{R}\left(\boldsymbol{b} ; \zeta_{B, f}, \mu_{f}, \zeta_{B, i}, \mu_{i}\right) \tilde{C}_{g g *}^{(1) i} \\
& \times\left(\zeta_{B}, \ln \frac{\sqrt{\zeta_{B, i}}}{q^{-}}, \eta_{a}, \boldsymbol{b}, \boldsymbol{k}, \mu_{i}, \bar{f}^{(1)}\right),
\end{aligned}
$$

with the evolution operator,

$$
\begin{aligned}
\tilde{R}\left(\boldsymbol{b} ; \zeta_{B, f}, \mu_{f}, \zeta_{B, i}, \mu_{i}\right)= & \exp \left\{\int_{\mu_{i}}^{\mu_{f}} \frac{d \bar{\mu}}{\bar{\mu}} \gamma_{G}\left(\alpha_{s}(\bar{\mu}), \ln \frac{\zeta_{B, f}}{\bar{\mu}^{2}}\right)\right\} \\
& \times\left(\frac{\zeta_{B, f}}{\zeta_{B, i}}\right)^{-\frac{\tilde{K}_{C S}\left(b, \mu_{i}\right)}{2}} .
\end{aligned}
$$

In the above expression, the CS kernel $\tilde{K}_{\mathrm{CS}}\left(\boldsymbol{b}, \mu_{i}\right)$ is in general assumed to have both perturbative and nonperturbative contributions; for a detailed discussion, see $[2,64,90]$. The nonperturbative contribution arises due to the inverse Fourier transform, which requires to integrate over large values of $\boldsymbol{b}$, well into the nonperturbative region. Note that a similar statement applies in principle for the convolution integral of Eq. (97), if the unintegrated gluon distribution does not dropoff sufficiently fast for small values of transverse momentum $\boldsymbol{k}$. The actual evolution takes place in two steps: first, one evolves the coefficient at a certain initial renormalization scale $\mu_{i}$ from an initial rapidity $y_{c, i}$-parametrized through $\zeta_{B, k}=\left(q^{-}\right)^{2} e^{2 y_{c, k}}$, 
$k=i, f$-to a final rapidity $y_{c, f}$. The second step evolves then the TMD PDF from the initial to the final renormalization scale. While the value of the final renormalization scale is of the order of the hard scale, i.e., the Higgs mass for the current example, the initial renormalization scale $\mu_{i}$ must be chosen such that it minimizes the perturbative correction to the TMD coefficients. In collinear calculations, it is naturally taken to be of the order of the transverse momentum $\boldsymbol{q}$ or its inverse conjugate coordinate $\mu_{b}=$ $2 e^{-2 \gamma_{E}} /|\boldsymbol{b}|$ with $\gamma_{E} \simeq 0.577216$ the Euler constant. In the current setup, the optimal choice is far from apparent, since the coefficients depend on multiple scales due to nonzero initial transverse momenta. In particular, the transverse momenta $\boldsymbol{k}$ and $\boldsymbol{q}$ are at least at first not necessarily of the same order of magnitude.

\section{A. A comparison of the kernels of CS and BFKL evolution}

Both CS and BFKL evolution describe evolution in rapidity. It is therefore natural to expect that both evolution and their respective kernels have a certain overlap. For the derivation of the CS kernel, we follow closely [2,90], where the kernel of the Collins-Soper evolution equation is defined through the $y_{c}$ dependence of the renormalized soft factor,

$$
\tilde{K}_{\mathrm{CS}}(\boldsymbol{\xi}, \mu)=\frac{\partial}{\partial y_{c}} \ln \left[\tilde{\mathcal{S}}\left(\boldsymbol{\xi}, y_{c}, \mu\right) \mathcal{Z}_{G}\left(y_{c}\right)\right]
$$

and is itself subject to the following renormalization group equation:

$$
\begin{aligned}
\frac{d \tilde{K}_{\mathrm{CS}}}{d \ln \mu^{2}} & =-\Gamma_{\mathrm{cusp}}^{A}\left(\alpha_{s}(\mu)\right), \quad \Gamma_{\mathrm{cusp}}^{A}=\sum_{n=1}^{\infty}\left(\frac{\alpha_{s}}{4 \pi}\right)^{n} \Gamma_{n-1}^{A}, \\
\Gamma_{0}^{A} & =4 C_{A}, \quad \Gamma_{1}^{A}=\Gamma_{0}^{A}\left[\left(\frac{67}{9}-\frac{\pi^{2}}{3}\right) C_{A}-\frac{10 n_{f}}{9}\right],
\end{aligned}
$$

where $\Gamma_{\text {cusp }}^{A}$ is the cusp anomalous dimension in the adjoint representation, see $[64,91,92]$ for higher order terms. At one loop, one finds

$$
\tilde{K}_{\mathrm{CS}}^{(1)}(\boldsymbol{\xi}, \mu)=\frac{\alpha_{S} C_{A}}{\pi} \ln \left(\frac{4 e^{-2 \gamma_{E}}}{\xi^{2} \mu^{2}}\right) .
$$

While the representation in transverse coordinate space is very useful for a direct solution of the CS equation through exponentiation of the CS kernel as in Eq. (85), it is also instructive to formulate the CS equation in momentum space, which allows for a direct comparison with the BFKL equation. In particular, defining in complete analogy to the BFKL treatment a CS Green's function $G_{i}\left(\Delta y, \boldsymbol{k}_{1}, \boldsymbol{k}_{2}\right)$, $i=\mathrm{BFKL}, \mathrm{CS}$, such that

$$
\begin{aligned}
G_{i}\left(0, \boldsymbol{k}_{1}, \boldsymbol{k}_{2}\right) & =\delta^{(2)}\left(\boldsymbol{k}_{1}-\boldsymbol{k}_{2}\right), \quad i=\mathrm{BFKL}, \mathrm{CS} \\
\frac{d G_{i}\left(\Delta y, \boldsymbol{k}_{1}, \boldsymbol{k}_{2}\right)}{d \Delta y} & =\int \frac{d^{2} \boldsymbol{k}}{\pi} K_{i}\left(\boldsymbol{k}_{1}, \boldsymbol{k}\right) G_{i}\left(\Delta y, \boldsymbol{k}, \boldsymbol{k}_{2}\right),
\end{aligned}
$$

one finds at one loop the following simple relation between both kernels:

$$
\begin{aligned}
K_{\mathrm{CS}}^{(1)}\left(\boldsymbol{k}_{1}, \boldsymbol{k}_{2}, \mu\right)= & K_{\mathrm{BFK}}^{(1)}\left(\boldsymbol{k}_{1}, \boldsymbol{k}_{2}\right) \\
& -\frac{\alpha_{S} C_{A}}{\pi} \delta^{(2)}\left(\boldsymbol{k}_{1}-\boldsymbol{k}_{2}\right) \ln \frac{\mu^{2}}{\boldsymbol{k}_{1}^{2}} .
\end{aligned}
$$

The presence of this factor can be explained as follows. As it is well-known, the virtual correction to the one-loop BFKL kernel is directly related to the gluon Regge trajectory $\omega(\epsilon, \boldsymbol{k})$, which in transverse momentum space can be written as

$$
\begin{aligned}
\omega\left(\epsilon, \boldsymbol{k}^{2}\right) & =-\frac{\alpha_{s} C_{A}}{4 \pi \mu^{2 \epsilon} \Gamma(1-\epsilon)} \int \frac{d^{2+2 \epsilon} \boldsymbol{l}}{\pi^{1+\epsilon}} \frac{\boldsymbol{k}^{2}}{\boldsymbol{l}^{2}(\boldsymbol{k}-\boldsymbol{l})^{2}} \\
& =-\frac{\alpha_{s} C_{A}}{2 \pi} \frac{1}{\epsilon}\left(\frac{\boldsymbol{k}^{2}}{\mu^{2}}\right)^{\epsilon} .
\end{aligned}
$$

The CS equation is on the other hand limited to soft radiation, which implies restriction to transverse momenta $|\boldsymbol{l}| \ll|\boldsymbol{k}|$ and $|\boldsymbol{l}-\boldsymbol{k}| \ll|\boldsymbol{k}|$. The integrand in the above expression therefore reduces to

$$
\frac{\boldsymbol{k}^{2}}{\boldsymbol{l}^{2}(\boldsymbol{k}-\boldsymbol{l})^{2}} \simeq \frac{1}{\boldsymbol{l}^{2}}+\frac{1}{(\boldsymbol{l}-\boldsymbol{k})^{2}}
$$

and the integral vanishes within dimensional regularization through a cancellation of the infrared and ultraviolet $1 / \epsilon$ poles. Removing on the other hand, the UV pole through renormalization, one finally ends up with the virtual contribution to the CS kernel. The CS evolution can be therefore understood as the soft approximation to the complete BFKL kernel. Indeed, such an identification is natural, since the CS kernel arises from the rapidity divergence of the soft function, while the BFKL kernel from the rapidity divergence of partonic cross sections in the high energy limit $\sqrt{s} \rightarrow \infty$.

The above discussion clearly suggests that the CS and BFKL evolution are closely related to each other, with the CS kernel as the soft limit of the complete BFKL kernel. When considering CS and BFKL evolution for the same quantity, it is therefore necessary to remove the overlap of both evolution equations or alternatively, to restrict them to distinct regions in phase space, which are then covered by the regarding evolution equation. In particular, the various evolution parameters must obey the following ordering:

$$
y_{c}^{f}>y_{c}^{i}=\eta_{a}>\eta_{b}
$$


it is needed to separate the phase space covered by BFKL evolution (rapidity evolution of the entire cross section) and CS evolution (rapidity evolution of soft gluons only). Clearly, soft gluons form a subset of the complete cross section and cannot be evolved separately from the latter in rapidity.

\section{B. $k_{T}$ factorization and alternative schemes}

In the following, we investigate our result for a specific choice of the evolution parameter of the unintegrated gluon density, which we fix to coincide with the proton momentum fraction $x$; i.e., we consider now the equivalent of
Eq. (49), but with the following choices for the parameters of the high energy evolution, following the results of Sec. III C:

$$
\begin{aligned}
\Delta \eta_{a b} & =\ln \frac{x_{0}}{x}, \quad \eta_{a}=\ln \frac{x_{0} M}{q^{-}}, \quad \eta_{b}=\ln \frac{M}{p_{b}^{-}}, \\
\bar{f}^{(1)}(\boldsymbol{q}, \boldsymbol{k}) & =\ln \left(\frac{x_{0} M}{|\boldsymbol{q}|}\right) \frac{1}{\pi}\left[\frac{1}{(\boldsymbol{q}-\boldsymbol{k})^{2}}\right]_{+},
\end{aligned}
$$

where $M$ is a still unspecified reference scale. The coefficients take the following form:

$$
\begin{aligned}
\hat{C}_{g g *}^{(1) f, k_{T}}\left(x_{0}, \zeta_{B} ; \boldsymbol{q}, \boldsymbol{k}, \mu\right)= & \frac{\alpha_{s} C_{A}}{2 \pi}\left\{\delta^{(2)}(\boldsymbol{l})\left[\ln \frac{\boldsymbol{q}^{2}}{\mu^{2}}\left(\ln \frac{\zeta_{B}}{\mu^{2}}-\frac{\beta_{0}}{2 C_{A}}\right)-\frac{1}{2} \ln ^{2} \frac{\boldsymbol{q}^{2}}{\mu^{2}}+\frac{67}{18}-\frac{5 n_{f}}{9 C_{A}}-\frac{\pi^{2}}{3}\right]+\ln \left(\frac{\zeta_{B}}{x_{0} \boldsymbol{q}^{2}}\right) \frac{1}{\pi}\left[\frac{1}{\boldsymbol{l}^{2}}\right]_{+}\right. \\
& \left.+\frac{1}{\pi} \int_{0}^{1} d z\left[\frac{z(1-z)\left(\boldsymbol{l}^{2}-\boldsymbol{q}^{2}\right)^{2}}{\left[z \boldsymbol{l}^{2}+(1-z) \boldsymbol{q}^{2}\right]^{2} \boldsymbol{k}^{2}}+\frac{1}{\boldsymbol{l}^{2}} \frac{\boldsymbol{k}^{2}-3(\boldsymbol{q}-\boldsymbol{k})^{2}-\boldsymbol{q}^{2}}{\left[z(\boldsymbol{q}-\boldsymbol{k})^{2}+(1-z) \boldsymbol{q}^{2}\right]}\right]\right\}, \\
\hat{C}_{g g *}^{(1) h, k_{T}}\left(x_{0}, \zeta_{B} ; \boldsymbol{q}, \boldsymbol{k}, \mu\right)= & \frac{\alpha_{s} C_{A}}{2 \pi}\left\{\delta^{(2)}(\boldsymbol{l})\left[\ln \frac{\boldsymbol{q}^{2}}{\mu^{2}}\left[\ln \frac{\zeta_{B}}{\mu^{2}}-\frac{\beta_{0}}{2 C_{A}}\right]-\frac{1}{2} \ln ^{2} \frac{\boldsymbol{q}^{2}}{\mu^{2}}+\frac{67}{18}-\frac{5 n_{f}}{9 C_{A}}-\frac{\pi^{2}}{3}\right]+\ln \left(\frac{\zeta_{B}}{x_{0} \boldsymbol{q}^{2}}\right) \frac{1}{\pi}\left[\frac{1}{\boldsymbol{l}^{2}}\right]_{+}\right. \\
& \left.+\ln \left(\frac{\zeta_{B}}{\boldsymbol{l}^{2}}\right) \frac{2\left((\boldsymbol{l} \cdot \boldsymbol{q})^{2}-\boldsymbol{l}^{2} \boldsymbol{q}^{2}\right)}{\pi \boldsymbol{l}^{2} \boldsymbol{q}^{2} \boldsymbol{k}^{2}}+\frac{1}{\pi} \int_{0}^{1} d z \frac{\left(\boldsymbol{q}^{2}-\boldsymbol{l}^{2}\right) 2 \boldsymbol{k} \cdot \boldsymbol{l}}{\left[z \boldsymbol{l}^{2}+(1-z) \boldsymbol{q}^{2}\right] \boldsymbol{l}^{2} \boldsymbol{k}^{2}}\right\},
\end{aligned}
$$

with $\boldsymbol{l}=\boldsymbol{k} \boldsymbol{- \boldsymbol { q }}$. Even though both $\eta_{a}$ and the function $\bar{f}^{(1)}$ depend within this scheme on a certain reference scale $M$, the dependence on this scale cancels between both contributions, and we remain only with the parameter $x_{0}$ which is of order one. The scale $M$ remains therefore unspecified and can be used to satisfy the ordering condition Eq. (93). A possible and suitable choice is then $\zeta_{B}^{i}=C \cdot \boldsymbol{q}^{2}$, with $C$ another constant of order one, which eliminates a potential large logarithm in the coefficients and which specifies eventually $M=|\boldsymbol{q}|$. The complete resummed gluon TMDs take then the following final form:

$$
\begin{aligned}
f_{g}^{k_{T}}\left(x, \zeta_{B, f}, \boldsymbol{q}, \mu_{f}\right)= & \exp \left\{\int_{\mu_{i}}^{\mu_{f}} \frac{d \bar{\mu}}{\bar{\mu}} \gamma_{G}\left(\alpha_{s}(\bar{\mu}), \ln \frac{\zeta_{B, f}}{\bar{\mu}^{2}}\right)\right\} \cdot \int d^{2} \boldsymbol{q}^{\prime} G_{\mathrm{CS}}\left(\frac{1}{2} \ln \frac{\zeta_{B, f}}{M^{2}}, \boldsymbol{q}, \boldsymbol{q}^{\prime}, \mu_{i}\right) \\
& \cdot \int \frac{d^{2} \boldsymbol{k}}{\pi} C_{g g^{*}}^{f, k_{T}}\left(x_{0}, M^{2}, \boldsymbol{q}^{\prime}, \boldsymbol{k}, \mu_{i}\right) \cdot \mathcal{G}^{k_{T}}\left(\ln \frac{x_{0}}{x}, \ln \frac{M}{p_{B}^{-}}, \boldsymbol{k}, \bar{f}_{k_{T}}^{(1)}\right), \\
h_{g}^{k_{T}}\left(x, \zeta_{B}, \boldsymbol{q}, \mu_{f}\right)= & \exp \left\{\int_{\mu_{i}}^{\mu_{f}} \frac{d \bar{\mu}}{\bar{\mu}} \gamma_{G}\left(\alpha_{s}(\bar{\mu}), \ln \frac{\zeta_{B, f}}{\bar{\mu}^{2}}\right)\right\} \cdot \int d^{2} \boldsymbol{q}^{\prime} G_{\mathrm{CS}}\left(\frac{1}{2} \ln \frac{\zeta_{B}^{f}}{M^{2}}, \boldsymbol{q}, \boldsymbol{q}^{\prime}, \mu_{i}\right) \\
& \times \int \frac{d^{2} \boldsymbol{k}}{\pi} C_{g g^{*}}^{h, k_{T}}\left(x_{0}, M^{2}, \boldsymbol{q}^{\prime}, \boldsymbol{k}, \mu\right) \mathcal{G}^{k_{T}}\left(\ln \frac{x_{0}}{x}, \ln \frac{M}{p_{B}^{-}}, \boldsymbol{k}, \bar{f}_{k_{T}}^{(1)}\right),
\end{aligned}
$$

where $\zeta_{B}^{i}=M^{2}$ and $\mu_{i}$ are yet unspecified scales. Reading the above expressions from the right to the left, one first evolves the unintegrated gluon density through BFKL evolution up to the hadron momentum fraction $x$, with a corresponding factorization uncertainty parametrized through $x_{0}$. The unintegrated gluon distribution is then convoluted with the NLO TMD coefficient in transverse momentum $\boldsymbol{k}$. The resulting expression defines then the gluon TMD with transverse momentum $\boldsymbol{q}^{\prime}$ at a scale $\zeta_{B}^{i}=$ $M^{2}$ and renormalization point $\mu_{i}$. While $M$ is an arbitrary scale, introduced to define the $k_{T}$-factorization scheme, it is naturally chosen to be of the order of the transverse momentum $\boldsymbol{q}^{\prime}$; the same is true for the choice of the renormalization point $\mu_{i}$. In a next step, it is therefore needed to evolve this gluon TMD both in $\zeta$ (rapidity evolution of soft gluons) and finally, in the renormalization scale to its final values, where rapidity evolution of solution gives rise to a convolution in transverse momentum $\boldsymbol{q}^{\prime}$. The above expressions for CSS evolution (combined evolution in $\zeta$ and $\mu$ ) are the conventional expresses found in the literature, while they are expressed in transverse momentum instead of transverse coordinate space. 
In particular, the CS Green's function in transverse momentum space is obtained from the frequently used transverse coordinate expression through

$$
G_{\mathrm{CS}}\left(\Delta y, \boldsymbol{q}, \boldsymbol{q}^{\prime}, \mu\right)=\int \frac{d^{2} \boldsymbol{b}}{(2 \pi)^{2}} e^{-i \boldsymbol{b} \cdot\left(\boldsymbol{q}_{t}-\boldsymbol{q}^{\prime}\right)} e^{\Delta y \cdot \tilde{K}_{\mathrm{CS}}(\boldsymbol{b}, \mu)} .
$$

At one loop, $\tilde{K}_{C S}$ is given by Eq. (86). For perturbative higher orders, see, e.g., Sec. III of [64], where it is needed to include a relative factor of 2 with respect to the convention employed in this paper. Apart from perturbative higher order corrections, one might also consider RG evolution from the scale $\mu_{i}$ to a suitable renormalization point of the CS Green's function; finally, it is also possible to include a model for nonperturbative effects. If one restricts oneself on the other hand to the leading order kernel Eq. (86), the above integral can be easily evaluated, and one finds

$$
\begin{aligned}
G_{\mathrm{CS}}^{\mathrm{LL}}\left(\Delta y, \boldsymbol{q}_{1}, \boldsymbol{q}_{2}, \mu\right)= & \frac{\Gamma\left(1-\bar{\alpha}_{s} \Delta y\right)}{\left(\boldsymbol{q}_{1}-\boldsymbol{q}_{2}\right)^{2} \Gamma\left(\bar{\alpha}_{s} \Delta y\right)} \\
& \times\left(\frac{\left(\boldsymbol{q}_{1}-\boldsymbol{q}_{2}\right)^{2} e^{-2 \gamma_{E}}}{\mu^{2}}\right)^{\bar{\alpha}_{s} \Delta y},
\end{aligned}
$$

with $\bar{\alpha}_{s}=\alpha_{s} C_{A} / \pi$. Note that convergence of the Fourier integral requires $\bar{\alpha}_{s} \Delta y<1$ for the above expression. While the kernels of CSS and BFKL evolution in Eq. (97) are well-known, our result provides as a new element the perturbative coefficient, which connects both evolution equations up to NLO accuracy. In particular, a complete next-to-leading logaritmic resummation of both BFKL and CSS logarithms requires to combine the NLO coefficient Eqs. (95), (96) with the unintegrated gluon distribution evolved with the NLO BFKL kernel [14] as well as CSS evolution with NLO anomalous dimension Eq. (80), and the corresponding expression for the CS kernel; see [64] for a compact summary up to NNLO accuracy of these elements in the gluonic channel. It would be very interesting to compare this result to the low $x$ expansion of exact $\mathrm{N}^{3} \mathrm{LO}$ results for the gluon TMD PDFs [93]. From a technical point of view, this would require to construct a partonic unintegrated gluon distribtion, following Eq. (43), but using NLO quark and gluon impact factors.

While the identification of the evolution parameter according to the $k_{T}$ scheme, as used above, provides a direct generalization of the collinear result and is been often employed in fits of the unintegrated gluon density, see e.g., [94-97], it is not necessarily the most adequate to describe rapidity evolution of the system. An alternative form would be to identify $\eta_{a}$ with the maximal rapidity of the soft gluonic system, $\eta_{a}=y_{c}$ or with the rapidity of the hard final state, i.e., the Higgs boson $\eta_{a}=y_{H}$. While $\eta_{a}=y_{c}$ eliminates entirely the need for $\mathrm{CS}$ evolution, it also ignores the rapidity of the hard event (Higgs boson) in the energy evolution of the TMD PDF. The choice appears therefore to be possible, but inadequate. The choice $\eta_{a}=y_{H}$ evolves the unintegrated gluon density through BFKL evolution up to the rapidity of the hard even, while CS evolution addresses the possible differences $y_{c}-y_{H}$, where both $y_{c}>y_{H}$ and $y_{c}<y_{H}$ is possible. Introducing furthermore the parameter $\delta y$, which allows us to address the scale uncertainty associated with high energy factorization; i.e., high energy evolution describes dependence on $\bar{y}_{H}=y_{H}+$ $\delta y$ and $e^{ \pm \delta y}$ is taken to be of order one, one finds

$$
\begin{aligned}
f_{g}^{\mathrm{rap}}\left(\bar{y}_{H}, y_{c}, \boldsymbol{q}, \mu\right)= & \exp \left\{\int_{\mu_{i}}^{\mu_{f}} \frac{d \bar{\mu}}{\bar{\mu}} \gamma_{G}\left(\alpha_{s}(\bar{\mu}), \ln \frac{\zeta_{B, f}}{\bar{\mu}^{2}}\right)\right\} \cdot \int d^{2} \boldsymbol{q}^{\prime} G_{\mathrm{CS}}\left(y_{c}-\bar{y}_{H}, \boldsymbol{q}, \boldsymbol{q}^{\prime}, \mu_{i}\right) \\
& \cdot \int \frac{d^{2} \boldsymbol{k}}{\pi} C_{g g^{*}}^{f}\left(\zeta_{B}^{i}, y_{c}, \bar{y}_{H}, \boldsymbol{q}, \boldsymbol{k}, \mu, 0\right) \cdot \mathcal{G}\left(\bar{y}_{H}-y_{0}, y_{0}, \boldsymbol{k}, 0\right), \\
h_{g}^{\text {rap. }}\left(\bar{y}_{H}, y_{c}, \boldsymbol{q}, \mu\right)= & \exp \left\{\int_{\mu_{i}}^{\mu_{f}} \frac{d \bar{\mu}}{\bar{\mu}} \gamma_{G}\left(\alpha_{s}(\bar{\mu}), \ln \frac{\zeta_{B, f}}{\bar{\mu}^{2}}\right)\right\} \cdot \int d^{2} \boldsymbol{q}^{\prime} G_{\mathrm{CS}}\left(y_{c}-\bar{y}_{H}, \boldsymbol{q}, \boldsymbol{q}^{\prime}, \mu_{i}\right) \\
& \cdot \int \frac{d^{2} \boldsymbol{k}}{\pi} C_{g g^{*}}^{h}\left(\zeta_{B}^{i}, y_{c}, \bar{y}_{H}, \boldsymbol{q}, \boldsymbol{k}, \mu, 0\right) \cdot \mathcal{G}\left(\bar{y}_{H}-y_{0}, y_{0}, \boldsymbol{k}, 0\right),
\end{aligned}
$$

where $\zeta_{B}^{i}=\left(M_{H}^{2}+\boldsymbol{q}^{2}\right) e^{-2 \delta y}, \zeta_{B}^{f}=\left(M_{H}^{2}+\boldsymbol{q}^{2}\right) e^{2\left(\bar{y}_{H}-y_{c}\right)}$ and $y_{0}$ is a parameter of the order of the hadron rapidity. Note that within this frame, the TMD PDFs no longer depend on the hadron momentum fraction, but rather on rapidity. While this might appear strange at first sight, it is natural from the point of view of high energy factorization where the momentum fraction is-in the case of the $k_{T}$-factorization schememerely an evolution parameter fixed through the kinematics of the final state, while the above rapidity scheme uses a different choice for this evolution parameter.

\section{Relation to previous results in the literature}

Before we conclude, we briefly discuss the relation of the above results to results in the literature. In principle, the TMD gluon distribution has been already study within high 
energy factorization at NLO in [22], previous studies with a similar scope are [20] and [98,99]. At the level of real corrections, the contributions seem to be identical at the level of Feynman diagrams, leaving aside the absence of the multiple reggeized gluon exchange in the current discussion. The set of virtual diagrams of [22] is on the other hand clearly reduced with respect to the ones considered in this work, Fig. 5. Indeed, the authors of [22] seem to consider only self-energy corrections to the Wilson line in their approach. This difference is directly related to the fact that [22] makes use of the so-called "shock wave picture" for the calculation of next-to-leading order corrections. While this is a frequently used frame for the calculations within the CGCframework at both LO and NLO, it does not allow us to recover the term proportional to $\beta_{0}$ in the anomalous dimension Eq. (80), as already noted by the authors of [22]. We believe that this constitutes an important advantage of the framework of the high energy effective action, since it does not only allow us to recover the double-logarithmic contribution to CSS resummation, (i.e., the Sudakov form factor of [22]) but also its single logarithmic terms.

Another point in which we somehow differ with [22], see also the discussion in [20], is the statement that BFKL and CS evolution cover by default distinct regions of phase space. As outlined above, this problem does strictly speaking not occur, if the evolution variable of the gluon density is identified with the hadron momentum fraction. To clarify this point further and to put this into context with the discussion in [22], we consider in the following the combined $z \rightarrow 0$ and $z \rightarrow 1$ singularities of the real corrections of our one-loop result. Since the treatment is slightly more involved for the linearly polarized gluon TMD, Eq. (64), we focus in the following on the unpolarized case, Eq. (63). Removing regulators through taken the limits $\rho, \sigma \rightarrow \infty$, and keeping only singular terms, we find that the TMD splitting function reduces to

$$
\begin{aligned}
& \int_{0}^{1} d z \frac{1}{(\boldsymbol{q}-\boldsymbol{k})^{2}} \tilde{\boldsymbol{P}}_{g g, r}^{(0) f}(z, \boldsymbol{q} \boldsymbol{k}) \\
& \quad \simeq \frac{\alpha_{s} C_{A}}{\pi \mu^{2 \epsilon} \Gamma(1-\epsilon)} \frac{1}{(\boldsymbol{q}-\boldsymbol{k})^{2}} \int_{0}^{1} d z\left(\frac{1}{z}+\frac{1}{1-z}\right) .
\end{aligned}
$$

At first sight, the poles at $z=0$ and $z=1$ are therefore indeed well separated. With the rapidity of the produced gluon equal to $\eta_{l}=\ln \frac{|q-k| z}{(1-z) q^{-}}$, the above integral can be however rewritten as

$$
\begin{aligned}
& \int_{0}^{1} d z \frac{1}{(\boldsymbol{q}-\boldsymbol{k})^{2}} \tilde{P}_{g g, r}^{(0) f}(z, \boldsymbol{q} \boldsymbol{k}) \\
& \quad \simeq \frac{\alpha_{s} C_{A}}{\pi \mu^{2 \epsilon} \Gamma(1-\epsilon)} \frac{1}{(\boldsymbol{q}-\boldsymbol{k})^{2}} \lim _{\sigma, \rho \rightarrow \infty} \int_{-\rho / 2}^{\sigma / 2} d \eta_{l},
\end{aligned}
$$

where we re-inserted the previously removed regulators $\rho, \sigma$ as cut-offs on the rapidity integral. As for the real part of the one-loop BFKL kernel within the high energy effective action, see $[46,48,53]$ for an explicit construction, the above expression is proportional to an integral which extends over the entire range of rapidity. In contrast to the derivation of the BFKL kernel, the above integral is however split up into a "soft" and a "hard" part,

$$
\begin{aligned}
\int_{-\rho / 2}^{\sigma / 2} d \eta_{l} & =\int_{-\rho / 2}^{\eta_{a}} d \eta_{l}+\int_{\eta_{a}}^{\sigma / 2} d \eta_{l} \\
& =\left(\frac{\sigma}{2}-\eta_{a}\right)+\left(\eta_{a}+\frac{\rho}{2}\right),
\end{aligned}
$$

where the distinction into "soft" and "hard" is essentially achieved through the virtual corrections. As a consequence -while we somehow agree with [22] that both pieces are well separated-care is needed to avoid overlap between both contributions. In particular, a gluon with a certain fixed rapidity may be either counted as soft or hard but never as both. At the same time, "gaps" in rapidity should be avoided for a consistent and correct description.

\section{CONCLUSIONS AND OUTLOOK}

In this paper, we extended the framework established in [62] for next-to-leading order corrections within Lipatov's high energy effective action to the case where the transition function contains an additional finite contributions. Using this extension, we were able to address the special, but important case of impact factors that possess a strong hierarchy with respect to their transverse scales, as it is the case within the $k_{T}$-factorization setup. The latter is characterized by a impact factor with a hard scale, i.e., the off shell partonic coefficient, and a hadronic impact factor, characterized by transverse momenta in the nonperturbative domain. The resulting expression have been found to agree with existing results in the literature, which have been established through a study of multiparticle production amplitudes in the (quasi-)multi-Regge kinematics [80]. Establishing this formalism at NLO within the high energy effective action is the first key result of this paper.

Another key result is the determination of the next-toleading order corrections to the gluon TMD PDFs in high energy factorization, making use of the established formalism for the renormalization of matrix elements of reggeized gluon fields. While unsubtracted NLO result is subject to both rapidity divergences due to high energy factorization and rapidity divergences due to definition of the TMD PDF, the subtracted and renormalized coefficient is completely free of such divergencies. In particular, we stress that rapidity divergences related to the definition of the TMD PDF can be treated using the soft factor, established within a setup based on collinear factorization. The same observation applies to 
the treatment of ultraviolet divergences and their renormalization. While this behavior was to be expected and indeed constitutes a necessary requirement, it provides a nontrivial check on the correctness of our result. While we confirm earlier results in the literature, which state that unpolarized and linearly polarized gluon TMD agree with each other in the dilute, i.e., BFKL limit, we find that both distributions differ at NLO, which is directly related to the nontrivial tensor structure of the real NLO corrections.

As a next step, we clarified further the relation between BFKL and CS evolution and clarified that they describe both evolution of the system in rapidity, i.e., BFKL of the cross section and therefore, directly related to the hard final state, while CS evolution rapidity evolution of the soft system. Both evolution equation are therefore not independent, and care is needed to avoid overcounting. Unlike previous calculations based on the CGC framework, our study further enabled us to recover the finite term proportional to $\beta_{0}$ in the anomalous dimension of the TMD PDFs.

As an important side result of our study, we find that the real NLO contribution of the unpolarized gluon TMD yield precisely the off shell TMD gluon-to-gluon splitting function, determined in [34]. While [34] determined this splitting function from a diagrammatic approach-essentially requiring simultaneous fulfillment of collinear and high energy limit while imposing gauge invariant production vertices - the current study obtains the same result from the QCD operator definition of gluon TMDs. It therefore establishes an important link between both frameworks, which will be of importance to continue with these efforts. In particular, the current study provides a possibility to finally determine the still missing virtual corrections to these splitting kernels and to formulate corresponding evolution equations.

Apart from these efforts, future studies should investigate phenomenological consequences of the derived results, which now allow us to use the complete CSS resummation formulation to resum double and single logarithmic contributions with the methods of the renormalization group, extending previous results in the literature, such as $[22,27,98,99]$. Another direction of research should address the inclusion of high density effects, along the lines of [22], but including the complete treatment of factorization scheme dependence established in this paper, and well as contributions due to quarks.

\section{ACKNOWLEDGMENTS}

I would like to thank Aleksander Kusina, Krzysztof Kutak, and Mirko Serino for collaboration at an early stage of this project. I am also grateful to Krzysztof Kutak for his comments on the draft. Support by Consejo Nacional de Ciencia y Tecnología Grant No. A1 S-43940 (CONACYTSEP Ciencias Básicas) is gratefully acknowledged.
[1] R. Angeles-Martinez, A. Bacchetta, I. I. Balitsky, D. Boer, M. Boglione, R. Boussarie, F. A. Ceccopieri, I. O. Cher ednikov, P. Connor, M. G. Echevarria et al., Transverse Momentum Dependent (TMD) parton distribution functions: status and prospects, Acta Phys. Pol. B 46, 2501 (2015).

[2] J. Collins, Foundations of perturbative QCD, Cambridge Monogr. Part. Phys., Nucl. Phys., Cosmol. 32, 1 (2011).

[3] N. A. Abdulov, A. Bacchetta, S. Baranov, A. B. Martinez, V. Bertone, C. Bissolotti, V. Candelise, L. I. Estevez Banos, M. Bury, P. L. S. Connor et al., TMDlib 2 and TMDplotter: a platform for 3D hadron structure studies, Eur. Phys. J. C 81, 752 (2021).

[4] J. C. Collins and D. E. Soper, Parton distribution and decay functions, Nucl. Phys. B194, 445 (1982).

[5] J. C. Collins and D. E. Soper, Back-to-back jets in QCD, Nucl. Phys. B193, 381 (1981); B213, 545(E) (1983).

[6] J. C. Collins, D. E. Soper, and G. F. Sterman, Transverse momentum distribution in Drell-Yan pair and $\mathrm{W}$ and $\mathrm{Z}$ boson production, Nucl. Phys. B250, 199 (1985).

[7] S. Catani, M. Ciafaloni, and F. Hautmann, Gluon contributions to small $\mathrm{x}$ heavy flavor production, Phys. Lett. B 242, 97 (1990).
[8] S. Catani, M. Ciafaloni, and F. Hautmann, High-energy factorization and small $\mathrm{x}$ heavy flavor production, Nucl. Phys. B366, 135 (1991).

[9] S. Catani, M. Ciafaloni, and F. Hautmann, High-energy factorization in QCD and minimal subtraction scheme, Phys. Lett. B 307, 147 (1993).

[10] E. A. Kuraev, L. N. Lipatov, and V. S. Fadin, Multi-Re ggeon processes in the Yang-Mills theory, Sov. Phys. JETP 44, 443-450 (1976).

[11] L. N. Lipatov, Reggeization of the vector meson and the vacuum singularity in nonabelian gauge theories, Sov. J. Nucl. Phys. 23, 338 (1976).

[12] E. A. Kuraev, L. N. Lipatov, and V. S. Fadin, The pomeranchuk singularity in nonabelian gauge theories, Sov. Phys. JETP 45, 199 (1977).

[13] I. I. Balitsky and L. N. Lipatov, The pomeranchuk singularity in quantum chromodynamics, Sov. J. Nucl. Phys. 28, 822 (1978).

[14] V. S. Fadin and L. N. Lipatov, BFKL pomeron in the nextto-leading approximation, Phys. Lett. B 429, 127 (1998).

[15] M. Ciafaloni and G. Camici, Energy scale(s) and next-toleading BFKL equation, Phys. Lett. B 430, 349 (1998).

[16] L. V. Gribov, E. M. Levin, and M. G. Ryskin, Semihard processes in QCD, Phys. Rep. 100, 1 (1983). 
[17] F. Dominguez, C. Marquet, B. W. Xiao, and F. Yuan, Universality of unintegrated gluon distributions at small x, Phys. Rev. D 83, 105005 (2011).

[18] F. Dominguez, J. W. Qiu, B. W. Xiao, and F. Yuan, On the linearly polarized gluon distributions in the color dipole model, Phys. Rev. D 85, 045003 (2012).

[19] I. Balitsky and A. Tarasov, Rapidity evolution of gluon TMD from low to moderate $\mathrm{x}, \mathrm{J}$. High Energy Phys. 10 (2015) 017.

[20] J. Zhou, The evolution of the small x gluon TMD, J. High Energy Phys. 06 (2016) 151.

[21] C. Marquet, E. Petreska, and C. Roiesnel, Transversemomentum-dependent gluon distributions from JIMWLK evolution, J. High Energy Phys. 10 (2016) 065.

[22] B. W. Xiao, F. Yuan, and J. Zhou, Transverse momentum dependent parton distributions at small-x, Nucl. Phys. B921, 104 (2017).

[23] T. Altinoluk, R. Boussarie, and P. Kotko, Interplay of the CGC and TMD frameworks to all orders in kinematic twist, J. High Energy Phys. 05 (2019) 156.

[24] T. Altinoluk, C. Marquet, and P. Taels, Low-x improved TMD approach to the lepto- and hadroproduction of a heavy-quark pair, J. High Energy Phys. 06 (2021) 085.

[25] M. Nefedov, Sudakov resummation from BFKL, arXiv:2105.13915.

[26] A. Dumitru, V. Skokov, and T. Ullrich, Measuring the Weizsäcker-Williams distribution of linearly polarized gluons at an electron-ion collider through dijet azimuthal asymmetries, Phys. Rev. C 99, 015204 (2019).

[27] A. Stasto, S. Y. Wei, B. W. Xiao, and F. Yuan, On the dihadron angular correlations in forward $p A$ collisions, Phys. Lett. B 784, 301 (2018).

[28] H. Mäntysaari, N. Mueller, F. Salazar, and B. Schenke, Multigluon Correlations and Evidence of Saturation from Dijet Measurements at an Electron-Ion Collider, Phys. Rev. Lett. 124, 112301 (2020).

[29] A. van Hameren, P. Kotko, K. Kutak, and S. Sapeta, Sudakov effects in central-forward dijet production in high energy factorization, Phys. Lett. B 814, 136078 (2021).

[30] H. Fujii, C. Marquet, and K. Watanabe, Comparison of improved TMD and CGC frameworks in forward quark dijet production, J. High Energy Phys. 12 (2020) 181.

[31] R. Boussarie, H. Mäntysaari, F. Salazar, and B. Schenke, The importance of kinematic twists and genuine saturation effects in dijet production at the Electron-Ion Collider, arXiv:2106.11301.

[32] O. Gituliar, M. Hentschinski, and K. Kutak, Transversemomentum-dependent quark splitting functions in $k_{T^{-}}$ factorization: Real contributions, J. High Energy Phys. 01 (2016) 181.

[33] M. Hentschinski, A. Kusina, and K. Kutak, Transverse momentum dependent splitting functions at work: Quark-togluon splitting, Phys. Rev. D 94, 114013 (2016).

[34] M. Hentschinski, A. Kusina, K. Kutak, and M. Serino, TMD splitting functions in $k_{T}$ factorization: The real contribution to the gluon-to-gluon splitting, Eur. Phys. J. C 78, 174 (2018).

[35] S. Catani and F. Hautmann, High-energy factorization and small $\mathrm{x}$ deep inelastic scattering beyond leading order, Nucl. Phys. B427, 475 (1994).
[36] S. Catani and F. Hautmann, Quark anomalous dimensions at small x, Phys. Lett. B 315, 157 (1993).

[37] H. Jung, S. Baranov, M. Deak, A. Grebenyuk, F. Haut mann, M. Hentschinski, A. Knutsson, M. Kramer, K. Kutak, A. Lipatov et al., The CCFM Monte Carlo generator CASCADE version 2.2.03, Eur. Phys. J. C 70, 1237 (2010).

[38] F. Hautmann, M. Hentschinski, and H. Jung, Forward Z-boson production and the unintegrated sea quark density, Nucl. Phys. B865, 54 (2012).

[39] F. Hautmann, L. Keersmaekers, A. Lelek, and A. M. Van Kampen, Dynamical resolution scale in transverse momentum distributions at the LHC, Nucl. Phys. B949, 114795 (2019).

[40] A. Bermudez Martinez, P. Connor, D. Dominguez Damiani, L. I. Estevez Banos, F. Hautmann, H. Jung, J. Lidrych, M. Schmitz, S. Taheri Monfared, Q. Wang et al., Production of Z-bosons in the parton branching method, Phys. Rev. D 100, 074027 (2019).

[41] S. Baranov, A. Bermudez Martinez, L. I. Estevez Banos, F. Guzman, F. Hautmann, H. Jung, A. Lelek, J. Lidrych, A. Lipatov, M. Malyshev et al., CASCADE3 A Monte Carlo event generator based on TMDs, Eur. Phys. J. C 81, 425 (2021).

[42] M. Ciafaloni, Coherence Effects in Initial Jets at Small q**2/s, Nucl. Phys. B296, 49 (1988).

[43] S. Catani, F. Fiorani, and G. Marchesini, QCD coherence in initial state radiation, Phys. Lett. B 234, 339 (1990).

[44] L. N. Lipatov, Gauge invariant effective action for highenergy processes in QCD, Nucl. Phys. B452, 369 (1995).

[45] L. N. Lipatov, Small x physics in perturbative QCD, Phys. Rep. 286, 131 (1997).

[46] M. Hentschinski and A. Sabio Vera, NLO jet vertex from Lipatov's QCD effective action, Phys. Rev. D 85, 056006 (2012).

[47] M. Hentschinski, Pole prescription of higher order induced vertices in Lipatov's QCD effective action, Nucl. Phys. B859, 129 (2012).

[48] G. Chachamis, M. Hentschinski, J. D. Madrigal Martínez, and A. Sabio Vera, Next-to-leading order corrections to the gluon-induced forward jet vertex from the high energy effective action, Phys. Rev. D 87, 076009 (2013).

[49] G. Chachamis, M. Hentschinski, J. D. Madrigal Martinez, and A. Sabio Vera, Quark contribution to the gluon Regge trajectory at NLO from the high energy effective action, Nucl. Phys. B861, 133 (2012).

[50] G. Chachamis, M. Hentschinski, J. D. Madrigal Martinez, and A. Sabio Vera, Gluon Regge trajectory at two loops from Lipatov's high energy effective action, Nucl. Phys. B876, 453 (2013).

[51] M. Hentschinski, Color glass condensate formalism, BalitskyJIMWLK evolution, and Lipatov's high energy effective action, Phys. Rev. D 97, 114027 (2018).

[52] G. Chachamis, M. Hentschinski, J. D. Madrigal Martínez, and A. Sabio Vera, Forward jet production \& quantum corrections to the gluon Regge trajectory from Lipatov's high energy effective action, Phys. Part. Nucl. 45, 788 (2014).

[53] M. Hentschinski, Lipatov's QCD high energy effective action: Past and future, in From the Past to the Future 
The Legacy of Lev Lipatov, edited by J. Bartels, V. Fadin, E. Levin, A. Levin, V. Kim, and A. Sabio Vera (World Scientific, Singapore, 2021), https://www.worldscientific .com/worldscibooks/10.1142/12127.

[54] P. Kotko and A. M. Stasto, Wilson lines in the MHV action, J. High Energy Phys. 09 (2017) 047.

[55] S. Bondarenko, L. Lipatov, S. Pozdnyakov, and A. Prygarin, One loop light-cone QCD, effective action for reggeized gluons and QCD RFT calculus, Eur. Phys. J. C 77, 630 (2017).

[56] A. van Hameren, Calculating off-shell one-loop amplitudes for $k_{T}$-dependent factorization: A proof of concept, arXiv:1710.07609.

[57] S. Bondarenko and S. Pozdnyakov, S-matrix and productions amplitudes in high energy QCD, Phys. Lett. B 783, 207 (2018).

[58] M. A. Braun, Triple-pomeron amplitude in the effective action approach, Eur. Phys. J. C 80, 774 (2020).

[59] E. Blanco, A. van Hameren, P. Kotko, and K. Kutak, Allplus helicity off-shell gauge invariant multigluon amplitudes at one loop, J. High Energy Phys. 12 (2020) 158.

[60] M. Gómez Bock, M. Hentschinski, and A. Sabio Vera, An effective field theory approach for electroweak interactions in the high energy limit, Eur. Phys. J. C 80, 1193 (2020).

[61] S. Bondarenko, S. Pozdnyakov, and A. Prygarin, Unifying approaches: BK equation from the Lipatov's effective action, arXiv:2106.01677.

[62] M. Hentschinski, K. Kutak, and A. van Hameren, Forward Higgs production within high energy factorization in the heavy quark limit at next-to-leading order accuracy, Eur. Phys. J. C 81, 112 (2021); Eur. Phys. J. C 81, 262(E) (2021).

[63] X. d. Ji, J. P. Ma, and F. Yuan, Transverse-momentumdependent gluon distributions and semi-inclusive processes at hadron colliders, J. High Energy Phys. 07 (2005) 020.

[64] M. G. Echevarria, T. Kasemets, P. J. Mulders, and C. Pisano, QCD evolution of (un)polarized gluon TMDPDFs and the Higgs $q_{T}$-distribution, J. High Energy Phys. 07 (2015) 158; J. High Energy Phys. 05 (2017) 073(E).

[65] G. Curci, W. Furmanski, and R. Petronzio, Evolution of parton densities beyond leading order: The nonsinglet case, Nucl. Phys. B175, 27 (1980).

[66] V. Ahrens, T. Becher, M. Neubert, and L. L. Yang, Origin of the large perturbative corrections to Higgs production at hadron colliders, Phys. Rev. D 79, 033013 (2009).

[67] P. J. Mulders and J. Rodrigues, Transverse momentum dependence in gluon distribution and fragmentation functions, Phys. Rev. D 63, 094021 (2001).

[68] X. d. Ji, J. p. Ma, and F. Yuan, QCD factorization for semiinclusive deep-inelastic scattering at low transverse momentum, Phys. Rev. D 71, 034005 (2005).

[69] J. C. Collins and T. C. Rogers, Equality of two definitions for transverse momentum dependent parton distribution functions, Phys. Rev. D 87, 034018 (2013).

[70] M. G. Echevarría, A. Idilbi, and I. Scimemi, Soft and collinear factorization and transverse momentum dependent parton distribution functions, Phys. Lett. B 726, 795 (2013).

[71] Due to the regulator defined in Sec. III D this implies in our case also an imaginary part; the above expressions refer to the corresponding real parts, while imaginary parts cancel for the final result.
[72] S. Cali, K. Cichy, P. Korcyl, P. Kotko, K. Kutak, and C. Marquet, On systematic effects in the numerical solutions of the JIMWLK equation, arXiv:2104.14254.

[73] L. N. Lipatov and M. I. Vyazovsky, QuasimultiRegge processes with a quark exchange in the $t$ channel, Nucl. Phys. B597, 399 (2001).

[74] M. Nefedov and V. Saleev, On the one-loop calculations with Reggeized quarks, Mod. Phys. Lett. A 32, 1750207 (2017).

[75] M. Nefedov and V. Saleev, Off-shell initial state effects, gauge invariance and angular distributions in the Drell-Yan process, Phys. Lett. B 790, 551 (2019).

[76] M. A. Nefedov, Computing one-loop corrections to effective vertices with two scales in the EFT for Multi-Regge processes in QCD, Nucl. Phys. B946, 114715 (2019).

[77] Note that the impact factors themselves might depend on additional transverse momenta; this is however irrelevant for the following discussion of high energy factorization and we therefore suppress this dependence in the following.

[78] M. Ciafaloni and D. Colferai, K factorization and impact factors at next-to-leading level, Nucl. Phys. B538, 187 (1999).

[79] This relation is exact for the scattering of a parton $a$ with a hadron $B$.

[80] J. Bartels, A. Sabio Vera, and F. Schwennsen, NLO inclusive jet production in $k_{T}$-factorization, J. High Energy Phys. 11 (2006) 051.

[81] T. Altinoluk and R. Boussarie, Low $x$ physics as an infinite twist (G)TMD framework: unravelling the origins of saturation, J. High Energy Phys. 10 (2019) 208.

[82] The overall factor arises since - at least at the level of bare distributions - the unintegrated gluon density reduces to the collinear gluon in the double logarithmic limit after integration over $\int d \boldsymbol{k}^{2}$ while the gluon TMD requires an integral over $\int d^{2} \boldsymbol{k}$, which gives rise to a relative factor of $\pi$.

[83] Note that this universality breaks down, once corrections due to multiple reggeized gluon exchange are included see e.g., [24].

[84] M.Hentschinski, J. D. M. Martínez, B. Murdaca, and A. Sabio Vera, The gluon-induced Mueller-Tang jet impact factor at next-to-leading order, Nucl. Phys. B889, 549 (2014).

[85] M. Hentschinski, J. D. Madrigal Martínez, B. Murdaca, and A. Sabio Vera, The quark induced Mueller-Tang jet impact factor at next-to-leading order, Nucl. Phys. B887, 309 (2014).

[86] M. Hentschinski, J. D. Madrigal Martínez, B. Murdaca, and A. Sabio Vera, The next-to-leading order vertex for a forward jet plus a rapidity gap at high energies, Phys. Lett. B 735, 168 (2014).

[87] S. Catani and M. H. Seymour, A general algorithm for calculating jet cross-sections in NLO QCD, Nucl. Phys. B485, 291 (1997); Nucl. Phys. B510, 503(E) (1998).

[88] See [69] and [70] for a detailed discussion of these limits.

[89] I. Z. Rothstein and I. W. Stewart, An effective field theory for forward scattering and factorization violation, J. High Energy Phys. 08 (2016) 025.

[90] S. M. Aybat and T. C. Rogers, TMD parton distribution and fragmentation functions with QCD evolution, Phys. Rev. D 83, 114042 (2011).

[91] A. Idilbi, X.d. Ji, J. P. Ma, and F. Yuan, Threshold resummation for Higgs production in effective field theory, Phys. Rev. D 73, 077501 (2006). 
[92] A. Idilbi, X.d. Ji, and F. Yuan, Transverse momentum distribution through soft-gluon resummation in effective field theory, Phys. Lett. B 625, 253 (2005).

[93] M. x. Luo, T. Z. Yang, H. X. Zhu, and Y. J. Zhu, Unpolarized quark and gluon TMD PDFs and FFs at $\mathrm{N}^{3} \mathrm{LO}$, J. High Energy Phys. 06 (2021) 115.

[94] M. Hentschinski, A. Sabio Vera, and C. Salas, Hard to Soft Pomeron Transition in Small-x Deep Inelastic Scattering Data Using Optimal Renormalization, Phys. Rev. Lett. 110, 041601 (2013).

[95] M. Hentschinski, A. Sabio Vera, and C. Salas, $F_{2}$ and $F_{L}$ at small $x$ using a collinearly improved BFKL resummation, Phys. Rev. D 87, 076005 (2013).
[96] G. Chachamis, M. Deák, M. Hentschinski, G. Rodrigo, and A. Sabio Vera, Single bottom quark production in $\mathrm{k}_{\perp}$-factorisation, J. High Energy Phys. 09 (2015) 123.

[97] F. G. Celiberto, Unraveling the unintegrated gluon distribution in the proton via $\rho$-meson leptoproduction, Nuovo Cimento C 42, 220 (2020).

[98] A. H. Mueller, B. W. Xiao, and F. Yuan, Sudakov Resummation in Small- $x$ Saturation Formalism, Phys. Rev. Lett. 110, 082301 (2013).

[99] A. H. Mueller, B. W. Xiao, and F. Yuan, Sudakov double logarithms resummation in hard processes in the small-X saturation formalism, Phys. Rev. D 88, 114010 (2013). 